\title{
Variation in Sitting Pressure Distribution and Location of the Points of Maximum Pressure with Rotation of the Pelvis, Gender and Body Characteristics
}

\author{
Niels C.C.M. Moes \\ Delft University of Technology \\ Delft, the Netherlands \\ Email: C.C.M.Moes@IO.TUDelft.nl \\ January 11, 2007
}

\begin{abstract}
The pressure distribution and the location of the points of maximum pressure, usually below the ischial tuberosities, was measured for subjects sitting on a flat, hard and horizontal support, and varying angle of the rotation of the pelvis. The pressure data were analyzed for force- and pressure-related quantities. Multiple regression was applied to explore relationships between these quantities and (i) a set of body characteristics, and (ii) the pelvis rotation. The maximum pressure and the pressure gradient were mainly explained by the ectomorphic index, and the distance between the maximum pressure points by gender and the angle of rotation of the pelvis.
\end{abstract}

Keywords: ergonomics, biomechanics, pelvis, pelvis rotation, pressure distribution, sitting, anthropometry, ischial tuberosity. 


\section{Introduction}

Design of sitting supports is often confronted with problems that relate to areas of high pressure and high pressure gradient. When such problems crop up discomfort is experienced, but the consequences can be more severe such as a reduction of concentration or even pressure sores. The approach to solving such problems is usually to apply an iterative series of adjustments on the shape of the support, possibly confirmed by measurements of the maximum pressure values. This paper reports the research on the pressure distribution for people sitting on a flat surface.

Since the shape of a seat is an important determinant for the experienced comfort designing the shape should be guided by ergonomics guidelines. In practice such guidelines are based on (i) physiological criteria, (ii) criteria from psycho-physical research (user assessment of comfort), and (iii) criteria that relate to the pressure distribution in the interface.

Since the force, that is exerted by the support, is transmitted via the contact area a pressure distribution exists, that induces a hydrostatic pressure distribution, an internal pressure gradient, and a shear stress distribution inside the body tissues. These effects contribute to several possible consequences by collapsing blood capillaries and lymph vessels, drainage of interstitial fluid, and a reduced functioning of nerve fibres (Romanus, 1976; Miller and Seale, 1985; Reddy et al., 1981a; Lundborg et al., 1983; Krouskop, 1983), possibly leading to medical complications. It is a generally adopted view that particularly the gradient of the hydrostatic pressure is a main indicator of decubitus.

Usually, although depending on the body posture, two areas of relatively high pressure exist below the seat bones (ischial tuberosities). In these regions the hydrostatic pressure and the shear stress are relatively high, so that blood capillaries, lymph vessels and nerve fibres are compressed, and interstitial fluid is drained to regions with lower pressure (Chow 
and Odell, 1978; Oomens et al., 1987; Levine et al., 1990; Staarink, 1995). The effect of externally exerted pressure on the interstitial fuid flow has been reported by Reddy et al. (1981b) and Reddy et al. (1981a). Krouskop (1983) discussed the effect of such drainage, leading to 'dried out' tissue, and cel and capillary damage. Inhibited blood flow may lead to a lack of oxygen supply, tissue starvation (necrosis) and pressure sores (decubitus) (Kosiak, 1961). If nerve fibres are under compressive load their ability to transport information may decrease (Rydevik and Nordborg, 1980; Dahlin et al., 1986).

The solution is to modify the shape of the seat by creating a hard surface of the right shape (Neff and Fischer, 1987; Brienza et al., 1996; Brienza and Karg, 1998), or by individually adapted reshaping (e.g., by using a cushion). Both methods are usually applied by trial and error, guided by the designer's knowledge and experience. The interface pressure can be used to control the shape modification (Souther et al., 1974; Mayo-Smith and Cochran, 1981; Brienza et al., 1996). Recently Brienza and Karg (1998) used an optimization algorithm for the shape of the seat using the stiffness distribution of the body tissues. They applied it for individual subjects and confirmed the improvement by pressure distribution measurements on foam supports that were manufactured for each subject.

The aim of the research is (i) to obtain the pressure distribution patterns of subjects seated on a flat, horizontal surface, (ii) to express the pressure distribution by a set of parameters that allow for a simple, physical interpretation, (iii) to statistically explain these parameters by a set of body characteristics, and (iv) to develop a predictive model of the pressure distribution.

This paper presents an overview of the literature about the seat pressure (section 1), the measurement setup to measure the pressure distribution, the body characteristics that are expected to correlate with the parameters of the pressure distribution (section 2), the 
mathematical models to analyse and parametrize the data (appendix), the measurement results and the analysis of the data (section 3), and a discussion of the results (section 4). When numerical results are given as $x x(y y)$, then $x x$ is the average and yy the sample standard deviation.

\section{Literature review}

Measurements of the sitting pressure distribution have been reported for various context, measurement devices, subjects, and research objectives. When the results of the different investigations are compared, it should be kept in mind that the applied measurement technique, device, and its underlying physical principles must be considered before drawing conclusions. Every measurement setup has its own emphasis, measurement procedure, circumstances and boundary conditions.

Only limited quantitative research was found on relationships between posture, body characteristics and pressure distribution. We intuitively assumed that the amount of subcutaneous fat and the muscle tissue below the ischial tuberosities reduce the maximum pressure by spreading the exerted force over a larger area. This is in concordance with the fact that typically ectomorphic people are more prone to painful sitting on a hard and flat chair than other people (Garber and Krouskop, 1982; Kernozek et al., 2000). The posture and the number of supporting surfaces have a significant influence on the pressure distribution parameters. For instance, bending the upper body backward or forward reduces or increases the foot supporting force, and using arm rests or a back rest reduces the force that is transmitted by the seat (sitting force), which can be proved using a simple biomechanical free body diagram. Zacharkow (1988), Minami et al. (1977), Helbig (1978) and Daniel and Faibisoff (1982) argued that in standing posture the ischial tuberosities are covered by 
muscular tissue, but that in upright sitting posture, when the hip is flexed, these muscular structures are shifted laterally so that only subcutaneous adipose tissue exists between the ischial tuberosity and the skin. In reclined sitting posture the body weight is transmitted to the seat mainly via the gluteal muscles, in upright posture via the ischial tuberosities, and in forward bended posture via the hamstring muscles.

Descriptors of pressure distribution The average pressure is defined as the ratio of the sitting force, and the size of the contact area. Low average pressures give a larger design space to redistribute a high pressure over regions of lower pressure, while for a high average pressures it is more difficult. Bader and Hawken (1986) and Maltais et al. (1999) found average pressure values from 6 to $10 \mathrm{kPa}$, but no relationship with posture, constitution or support characteristics was given. Aissaoui et al. (2001) found that the sitting force has a range of 240 to $410 \mathrm{~N}$, while the average size of the contact area was $770 \mathrm{~cm}^{2}$. Since the correspondence between the data of the sitting force and the contact area was missing, no conclusion can be drawn about the average pressure.

Obtaining maximum pressure values is complicated since the measuring elements have a finite size for pressure integration, and between the borders of neighbouring elements a gap exists. This has been neglected in most reports, that assume the maximum pressure equal to the maximum measured pressure. Reported values show a variation from $5 \mathrm{kPa}$ to $248 \mathrm{kPa}$ (Houle, 1969; Garber et al., 1978; Minns et al., 1984; Bader and Hawken, 1986; Henderson et al., 1994; Maltais et al., 1999; Aissaoui et al., 2001; Brienza and Karg, 1998; Brienza et al., 1996; Hobson, 1988; Riley and Bader, 1988). Highest values were found for disabled subjects sitting on a flat, hard support (Minns et al., 1984). Lowest values were found for healthy subjects, sitting on a curved support and using a backrest (Riley 
and Bader, 1988). Applying a $10^{\circ}$ reclined posture reduces the maximum pressure and the pressure gradient by $25 \%$ (Hobson, 1988). Henderson et al. (1994) found that a $35^{\circ}$ and a $65^{\circ}$ backward tilt of a wheelchair reduced the maximum pressure by $27 \%$ and $47 \%$. The highest maximum pressure reduction, 78\%, happened when the subject bended forward.

Stumbaum (1983) related the sitting force, the size of the contact area and the maximum pressure to weight, cushion and gender for healthy volunteers. Men showed a larger maximum pressure than women (difference $10 \mathrm{kPa}$ ), which can be explained by a larger average contact area for women (difference $50 \mathrm{~cm}^{2}$ ). The maximum pressure has a negative correlation with body mass ( $r=-0.37$ for men and 0.47 for women). Brienza and Karg (1998) found a negative correlation $\left(-0.58<r^{2}<-0.31\right)$ between the maximum pressure and the body mass index $\left(\mathrm{BMI}=\right.$ mass $/$ stature $\left.^{2}\right)$. The relationship between the maximum pressure and the type of body build (thin, average and obese) was investigated by Garber and Krouskop (1982) for disabled subjects. They found that the frequency of the occurrence of the maximum pressure below a bony area was 53\%, 35\% and $27 \%$ from thin to obese.

Gross et al. (1994) mentioned the relevance of the pressure gradient for seat comfort. Maltais et al. (1999), Brienza and Karg (1998), Hobson (1988) and Bader and Hawken (1986) found a range of $0.23 \mathrm{kPa} / \mathrm{m}$ to $6 \mathrm{kPa} / \mathrm{m}$ (higher values for the disabled). The pressure gradient was computed by linear interpolation, without considering the location.

Åkerblom (1948) found that the distance between the midpoints of the ischial tuberosities has a range of 9 to $15 \mathrm{~cm}$, where women show a larger distance than men. Other authors report a smaller range (Diffrient et al., 1981; Kira, 1976). Since the ischial bones converge towards the pubis, the distance of the high pressure areas should depend on a forward/backward rotation of the pelvis. However, Stumbaum (1983) found no significant 
dependency of the distance between the high pressure points on gender or on rotation of the backrest (not the pelvis); for upright sitting this distance was 121(14) mm.

\section{Methods and materials}

A cartesian coordinate system was used. The $x$-axis runs in lateral direction from right to left, the $y$-axis in backward sagittal direction and the $z$-axis in cranial (upward) direction. The origin is on the seat surface, in the mid-sagittal plane at the front edge of the support.

Three measuring methods and devices were used. The pressure distribution data and the location of the points of maximum pressure were obtained with the pressure distribution measuring device. The location of the points of maximum pressure (second measurement) and the shape and the size of the contact area were obtained using the mirror box which shows a low pressure threshold. The angle of the pelvis rotation was measured with the antenna method.

The pressure distribution measuring device The pressure distribution measuring device, figure 1a, is based on the capacitive method. It has an overall uncertainty of $10 \%$ of maximum load. The construction of the device and its calibration are reported in Moes (1999). It consists of a $24 \times 36$ rectangular array of $1 \mathrm{~cm}^{2}$ elements, spaced at $2 \mathrm{~mm}$ intervals, so that the distance between the centres of two neighbouring elements in a row or a column is $1.2 \mathrm{~cm}$. Its upper limit of calibration was $350 \mathrm{kPa}$. Values that exceed this maximum are set to $350 \mathrm{kPa}$. It is placed on top of a Kistler measuring platform that records the sitting force and the location of the line of action. A removable board is mounted for correct and reproduceble positioning of the subjects. Before the actual pressure distribution measurements it is removed so that the pelvis can rotate freely. 


\section{Pressure distribution and antenna measuring devices}

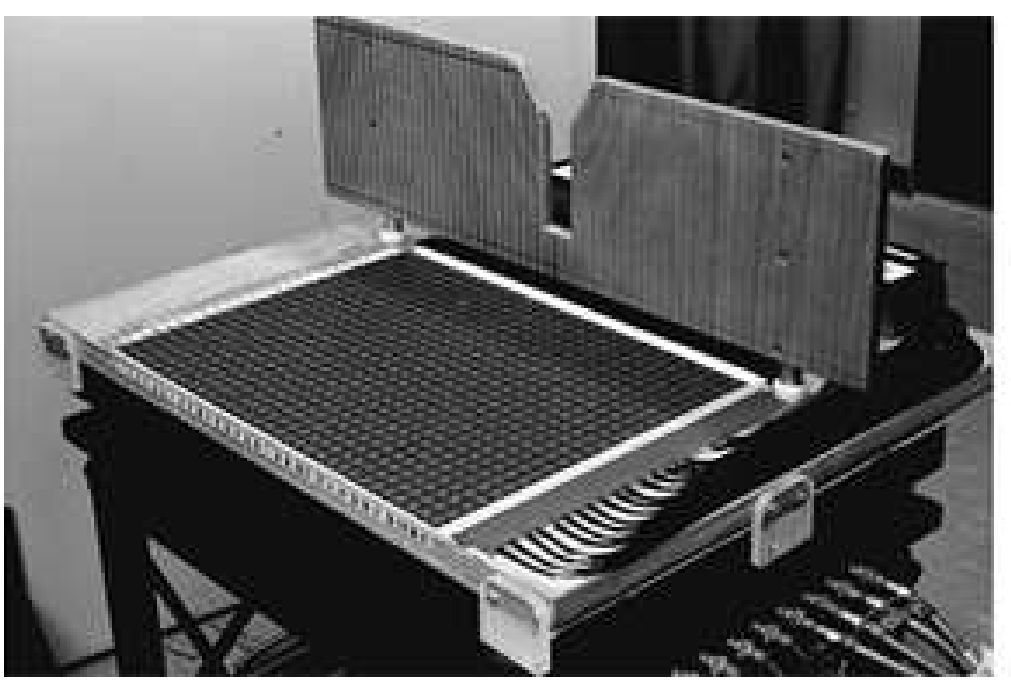

(a)

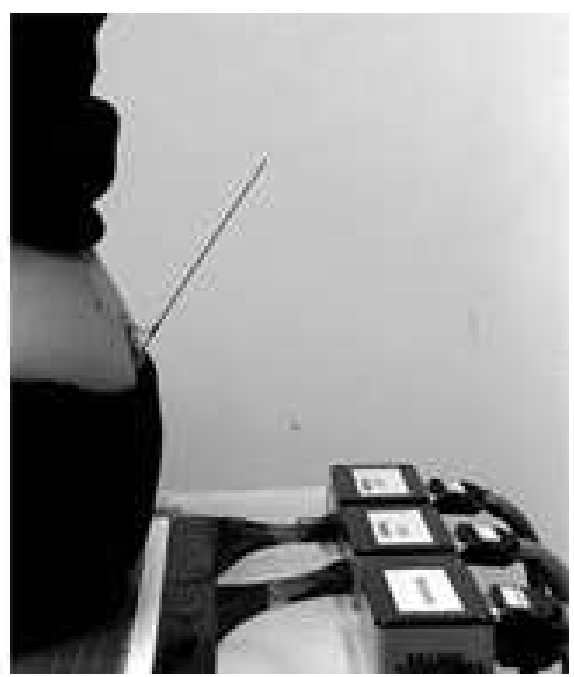

(b)

Figure 1: Figure a shows the pressure distribution measuring device, mounted on top of the Kistler platform. Figure $b$ shows the antenna arrangement for measuring the angle of the pelvis rotation.

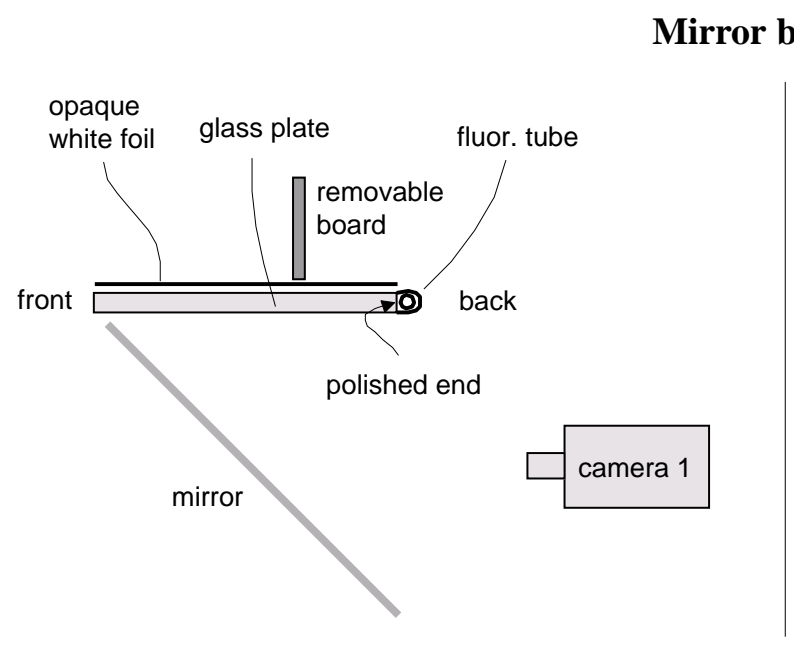

(a)

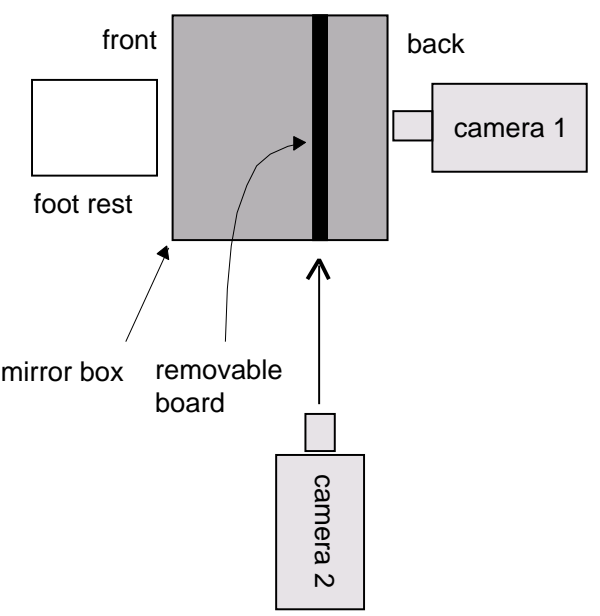

(b)

Figure 2: A lateral view of the mirror box (a) and a top view of the measurement setup for the mirror box (b). 


\section{Pressure distribution contour lines}

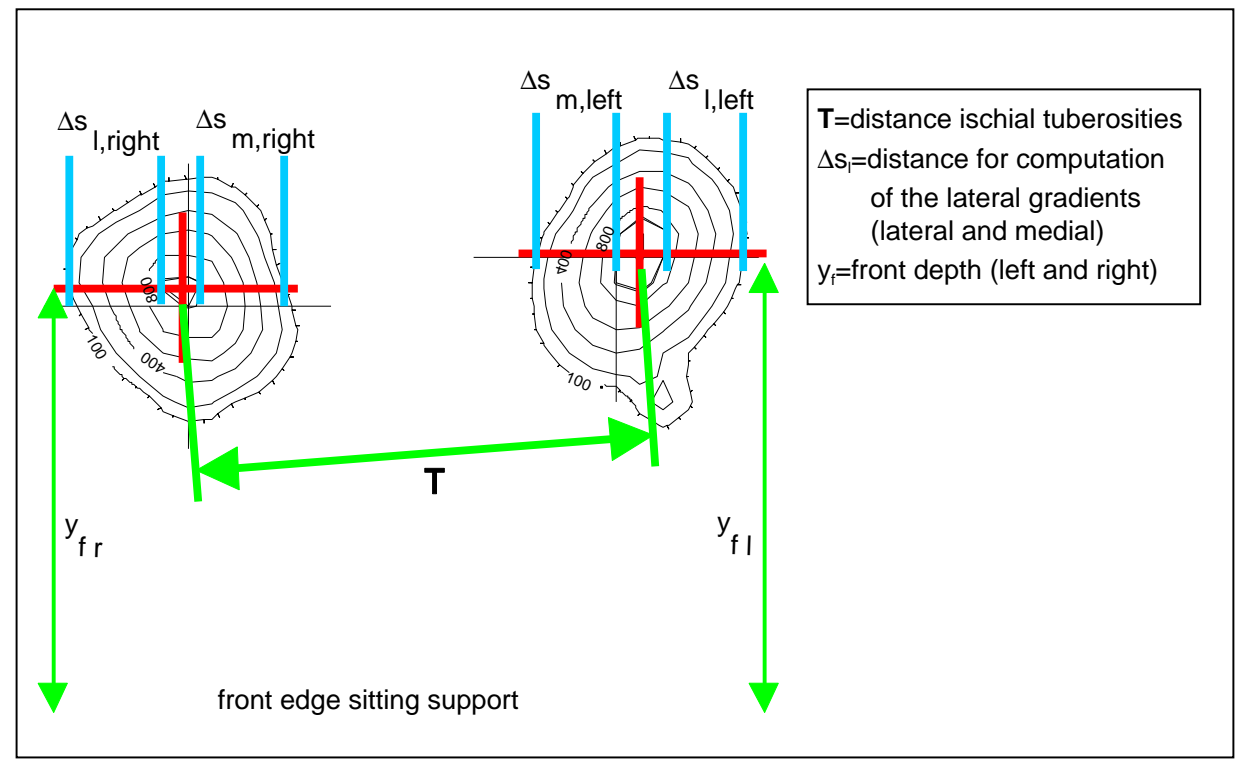

Figure 3: Example of a contourline diagram as it is generated by GPCP (General Purpose Contourline Package, implemented on Convex computer). 100 Units of the pressure scale corresponds to $10 \mathrm{kPa}$. The figure shows the distance between the ischial tuberosities, the regions for the computation of the lateral gradients, and the front depths.

The geometric centre of the highest pressure contourline (left and right) of the pressure distribution was considered the 'point' of maximum pressure, see figure 3. For the purpose of comparison, the locations of the maximum pressure areas were computed a second time based on the pressure distribution data.

The mirror box The measurement of the size of the contact area, as well as a second estimate of the location of the points of maximum pressure was done with the mirror box, a modified type of a paedobarograph, (Chodera and Lord, 1978; Treaster, 1987). It contains a $40 \times 40 \mathrm{~cm}$ glass plate of $1 \mathrm{~cm}$ thickness, figure $2 \mathrm{a}$. The sides of the glass plate are polished. One side is illuminated by a fluorescent tube. The light from the fluorescent tube enters the 
glass plate through the polished end and remains inside the glass since the incident angle exceeds the critical angle for total reflection. A mat of white opaque silicon rubber rubber, $1 \mathrm{~mm}$ thickness, covers the top of the glass plate. The lower side of this mat is roughly corrugated. If pressure is exerted at the top of the mat the contact between the white rubber and the glass is intensified, which modifies the ratio of the indices of refraction. Then the light enters the white rubber instead of being reflected so that the rubber foil is locally illuminated. A pressure of $0.1 \mathrm{kPa}$ was detected as a bright spot. Via the mirror the image of increased brightness is recorded by the camera 1. If the pressure increases, a larger area of the corrugations is pressed against the glass surface causing a corresponding increase of the size of the white spots, which is perceived as increased brightness. A top view of the measurement set up is shown schematically in figure $2 \mathrm{~b}$. The foot rest is a roller beared platform so that horizontal shear force is avoided. The removable board was used to position the subjects, then it was removed.

Antenna to measure the angle of pelvis rotation The angle of the pelvis rotation was measured according to the antenna method Moes (1998). A small rod (antenna) is mounted at the sacral skin, so that the pelvis rotation is reflected by the antenna rotation (figure 1b). Camera 2 records the rotation of the antenna. The angle of the pelvis rotation, $\alpha_{p}$, is computed from the angle of the antenna rotation, $\alpha_{a}$ with respect to the reference posture as: $\alpha_{p}=1.1 \times \alpha_{a}$. The standard deviation of the coefficient is $10 \%$. The reference posture is the posture of subjectively experienced maximum pressure at the skin below the ischial tuberosities. It is reproduced within $\pm 4^{\circ}$, see section 3 .

Measuring procedure The subjects wore a loosely fitting seamless legging over the bare skin. The following body characteristics were measured: the amount of subcutaneous fat 
(Durnin and Womersley, 1974), the somato type (endomorphic, mesomorphic and ectomorphic rating) (Carter and Heath, 1990), stature, body mass, the distance between the SIASes, the distance between the SIPSes ${ }^{1}$ and the thigh depth at the level of the buttock fold. Before the actual measurements took place, the subjects practised upright sitting and rotating the pelvis while keeping the body upright. The upright posture was not controlled by measurement. It turned out that keeping the upright posture was not difficult; correction by the research team was not needed.

The measurements were done in two sequences, the first on the mirror box, the second on the pressure distribution measuring device. The initial posture in each sequence was upright (the subjects experience maximum pressure under the seating bones, while the sacral skin touches the reference board). The line through the major trochanter and the lateral epicondyle of the femur was horizontal, the lower legs vertical. The distance between the knees was left free. This posture is called the reference posture, $\alpha_{p}=0^{\circ}$. Forward rotation is positive $\left(\alpha_{p}>0\right)$, and backward rotation negative $\left(\alpha_{p}<0\right)$. Then the board was removed. A measurement on the mirror box consists of the simultaneous recordings of the cameras. The second measurement series with the pressure distribution measuring device consists of the recordings of camera 2 for the angle of the pelvis rotation, and the pressure distribution data. The reference posture was the first posture for each series.

Then the subject rotated the pelvis stepwise from maximal backward to maximal forward while keeping the upper body upright, inclining the upper body neither backward nor forward, see figure 4 . Thus during each measuring cycle 10 posture measurements were done.

For each posture the pressure distribution and the contact area were measured. The

\footnotetext{
${ }^{1}$ Spina Iliaca Anterior Superior and Spina Iliaca Posterior Superior
} 


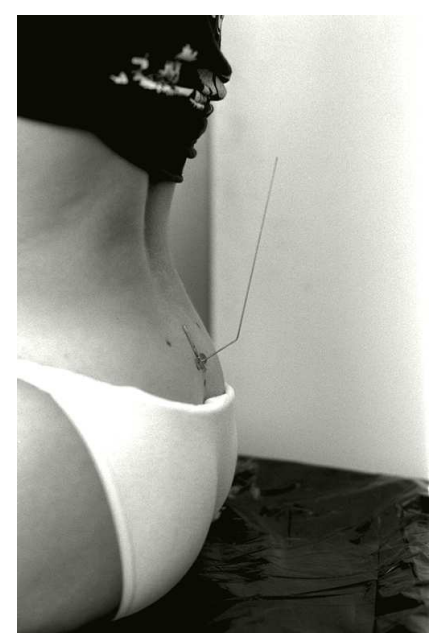

\section{Rotation of the pelvis}
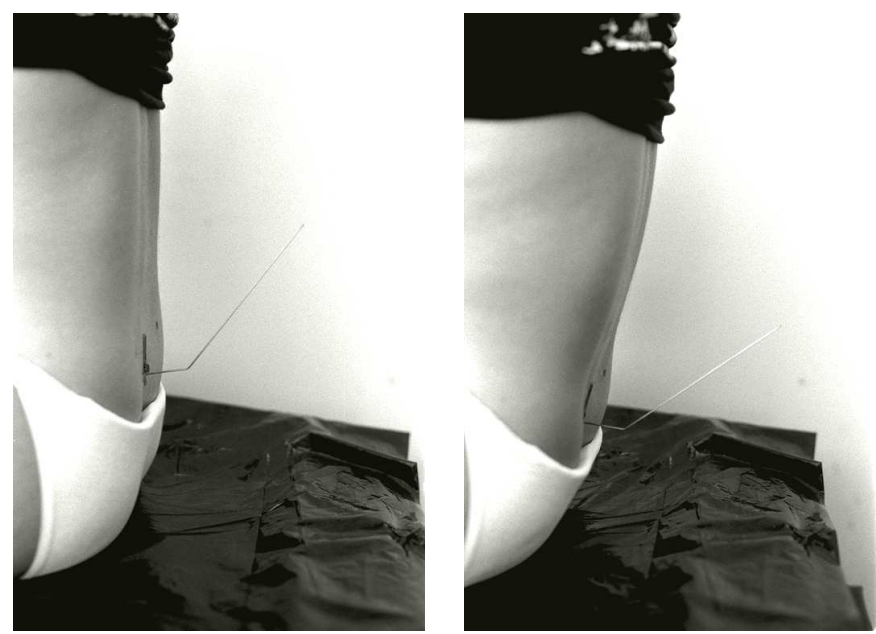

Figure 4: While the pelvis rotates, the upper body remains upright.

third posture was halfway the first two postures, the fourth posture was again the reference posture. The next postures were halfway forward, maximally forward and again halfway forward. The last three postures were again the reference posture, halfway backward and maximally backward. The time interval between the measurements on the pressure device was one minute to enable making corrections for hysteresis of the measuring device (Moes, 1999). During the one minute intervals the necessary readings were done with the current posture, the new posture was taken, and ca. 20 seconds were left before the next readings.

The reproducibility of the reference posture was defined as the standard deviation of the average reference posture. For each subject four measurement series were done: two series on the mirror box and two on the pressure distribution measuring device. On both devices one measurement series was done while the subject wore a loosely fitting legging, and one series with their own underwear. During each series the reference posture was taken three times, so that the four series resulted in twelve measurements for the reference posture.

The mathematical models to compute of the location of the maximum pressure points, 
Table 1: The body characteristics of the subjects.

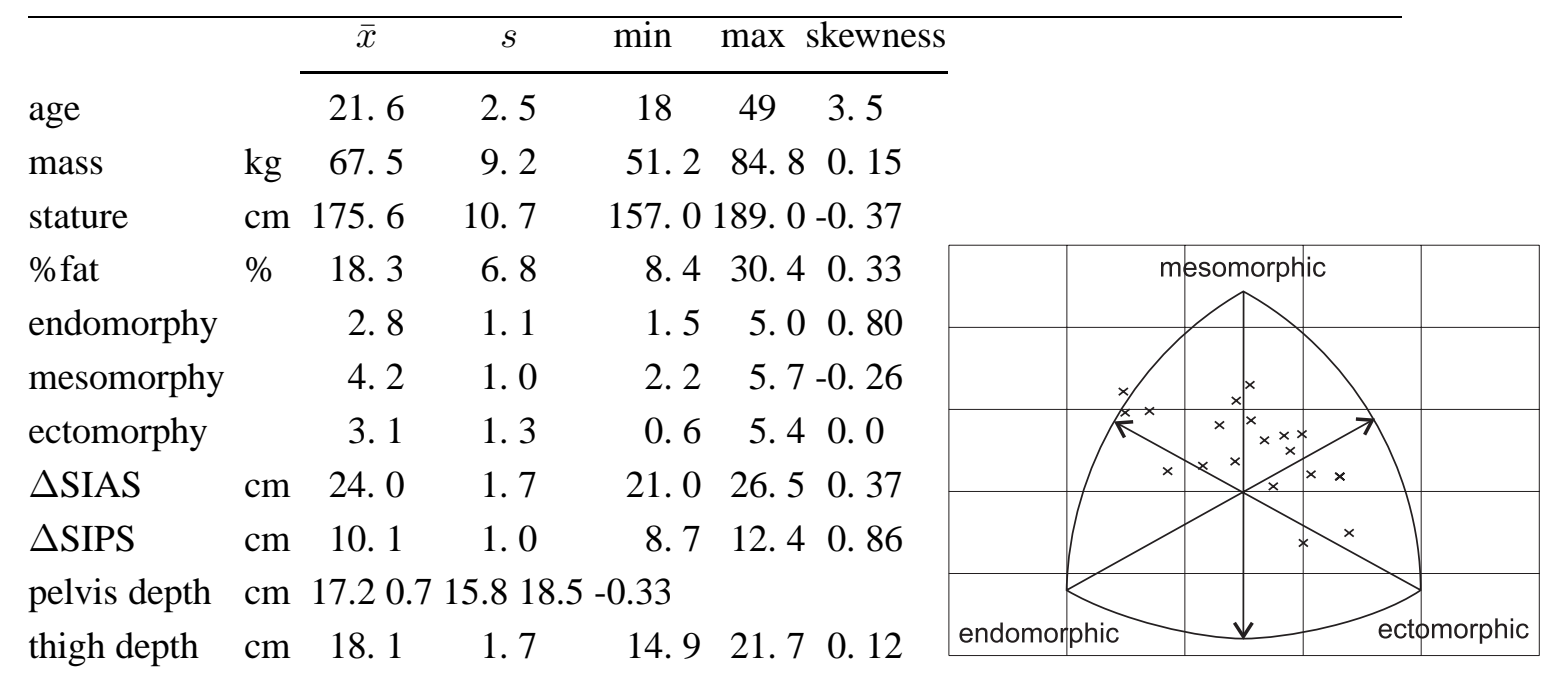

the pressure and te rotation parameters are explained in the appendix.

\section{Results}

This section gives the characteristics of the subjects, the pelvis rotation and related aspects, the location of the points of maximum pressure and the pressure distribution related quantities. The representation of the numerical results is the same as in the literature section: $x x(y y)$ means that $x x$ is the average and $y y$ the sample standard deviation. The derivative of the quantity $x$ to the pelvic angle, $\alpha_{p}$, is given by $\beta_{x}^{\alpha_{p}}=\partial x / \partial \alpha_{p}$. When gender is applied, zero (0) means female, and one 1) means male.

Subjects Eleven male and nine female subjects participated. The body characteristics are given in table 1. The high value of the skewness for age is caused by one subject being 
much older then the others. The figure at the right gives the distribution of the somato type of the subjects. The coordinates of the markers in this somatographical space were computed according to Carter and Heath (1990). The somatotypical characterizations vary between 1 and 8 (begin- and endpoints of the arrows). The pelvis depth, $s s_{a v g}$, was the sagittal distance between the midpoint of the SIASes and the SIPSes.

The reference posture and the pelvis rotation The reference posture (degrees) on the pressure distribution measuring device and the mirror box are related as: $\alpha_{m b}=0.96 \times$ $\alpha_{p d}+1.5 \quad(r=0.90)$. Apparently the angles of the reference posture show no significant difference for the two devices. For each subject the range of the reference posture was computed. The average range for all subjects was $7.3(3.4)^{\circ}$.

The average and the standard deviation of the maximum backward $\left(\alpha_{p}<0\right)$ and the maximum forward $\left(\alpha_{p}>0\right)$ rotation are not significantly different. Moreover, a significant relationship was not found, so that the ability to rotate forward does not predict the ability to rotate backward.

The reproducibility of the maximum rotation of the pelvis was derived from the comparison of maximum pelvis angle rotation on the mirror box device and the pressure distribution measurement device. In both measurement series the subjects received the same instructions. The coefficient of correlation is 0.97 , and the coefficient of regression 0.96 .

\subsection{Sagittal location}

The front depth ${ }^{2}$, in the reference posture is $y_{f}($ ref $)=17.7(2.2) \mathrm{cm}$ with minimum and maximum values of $12.4 \mathrm{~cm}$ and $21.6 \mathrm{~cm}$. This variability results from (i) the inaccuracy of

\footnotetext{
${ }^{2}$ The abolute value of the front depth has little meaning since it depends on the dimensions of the measurement set up. We focus on its changes.
} 
Front depth

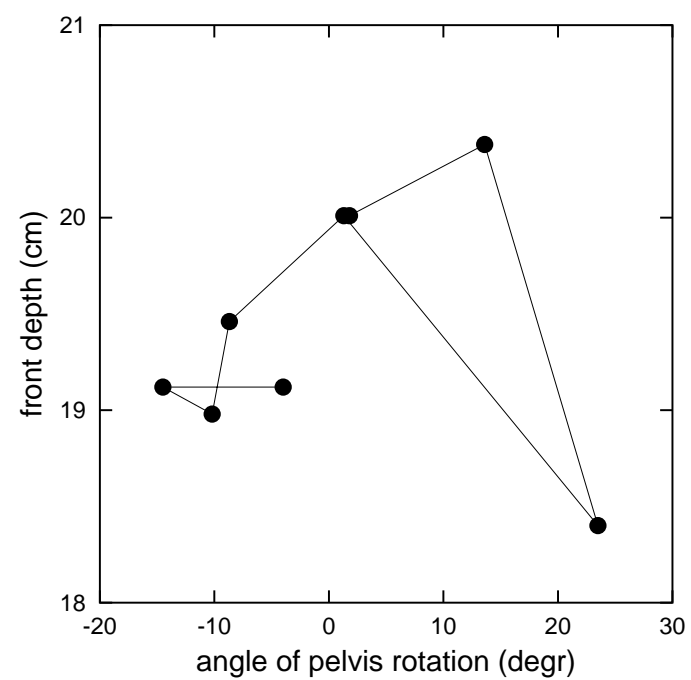

(a)

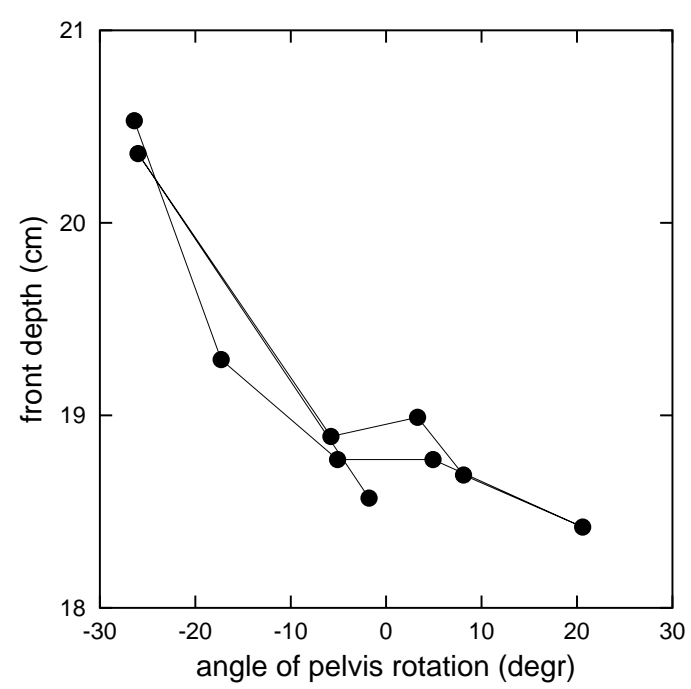

(c)

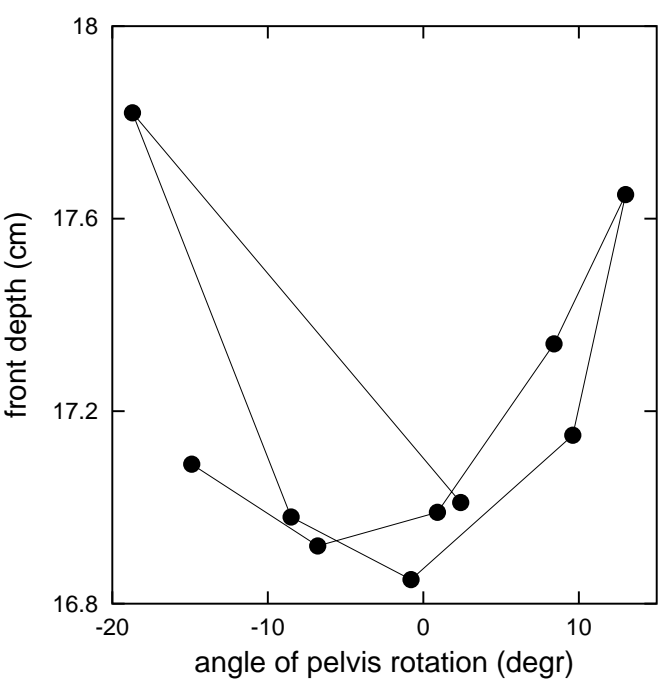

(b)

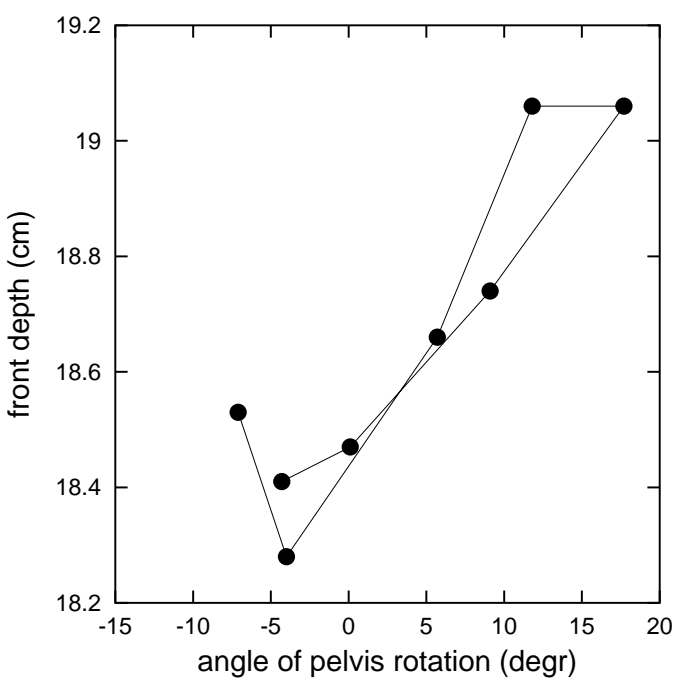

(d)

Figure 5: Four typical graphs showing $y_{f}$ against $\alpha_{p}$. Figures a to d give examples of the local maximum, the local minimum, the decreasing and the increasing behaviour of $y_{f}$. 
Table 2: The distance, $\mathrm{T}$, between the points of maximum pressure. $T_{0}$ is the distance in the reference posture. The abbreviation $m b$ and $p d$ refer to the mirror box device and the pressure distribution measuring device. The rows with $\beta^{\alpha_{p}}$ give the sensitivity of the distance for varying pelvis rotation.

\begin{tabular}{lrrrrrrrr}
\hline variable & & $\min$ & $\max$ & $\bar{x}$ & $s_{\bar{x}}$ & $s$ & $V$ & skew \\
\cline { 3 - 9 }$T_{0}(m b)$ & & & & & & & \\
$T_{0}(p d)$ & $(\mathrm{cm})$ & 10.3 & 15.0 & 12.3 & 0.4 & 1.5 & 0.12 & 0.34 \\
$T_{0}(\%, p d+m b)$ & $(\mathrm{cm})$ & 12.4 & 15.5 & 13.6 & 0.3 & 0.9 & 0.07 & \\
$T_{0}\left(\sigma^{7}, p d+m b\right)$ & $(\mathrm{cm})$ & 9.5 & 11.8 & 11.1 & 0.2 & 0.6 & 0.05 & \\
$\beta_{T(p d)}^{\alpha_{p}}$ & $(\mathrm{~cm} /$ degr $)$ & -0.077 & -0.009 & -0.038 & 0.004 & 0.016 & 0.42 & -0.32 \\
$\beta_{T(m b)}^{\alpha_{p}}$ & $(\mathrm{~cm} /$ degr $)$ & -0.098 & -0.035 & -0.054 & 0.005 & 0.021 & 0.39 & -1.28 \\
\hline
\end{tabular}

positioning a subject against the removable board, (ii) the inaccuracy of taking the reference posture, (iii) the thickness of living tissue between the dorsal surface of the sacrum and the skin surface, and (iv) the variability of the dimensions of the bony pelvis. Multiple regression showed no significant relationship of $y_{f}($ ref $)$ with the body characteristics. Analysis of $y_{f}\left(\alpha_{p}\right)$ showed no consistent relationship. Four typical relationships are shown in figures 5a-d. The first shows a local maximum $(n=2)$, the second a local minimum $(n=7)$, and the third and the fourth a monotonously decreasing $(n=2)$, respectively increasing $(n=7)$ function. Apparently the movement of the seating bones over the inner aspect of the skin consists of an unpredictable combination of rolling and sliding (see appendix).

Distance of the points of maximum pressure Table 2 summarises the results for the distance, $T$, of the points of maximum pressure. In the reference posture the average distance on the mirror box was $12.3(1.5) \mathrm{cm}$, and on the pressure device $12.4(1.5) \mathrm{cm}$. Comparison of $T_{0}$, obtained from the mirror box and the contourline data, gives $T_{0}(m b)=$ $0.995 \times T_{0}(p d)(r=1.0)$, which means that the two methods produce the same results for 


\section{Distance between ischial tuberosities for varying stature and pelvis rotation}

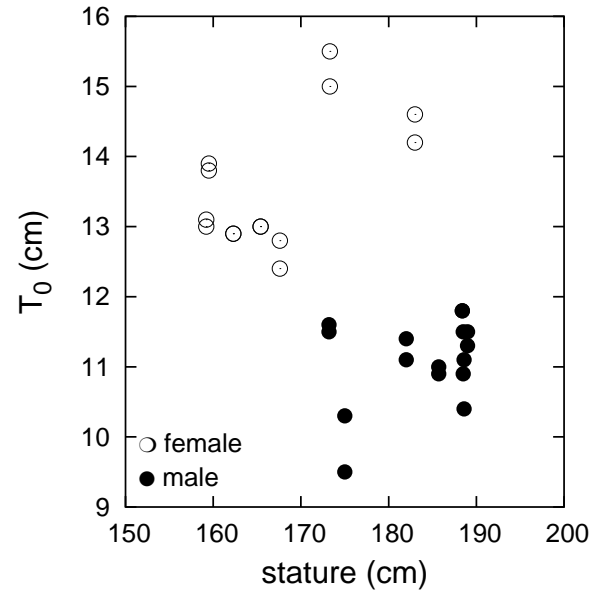

(a)

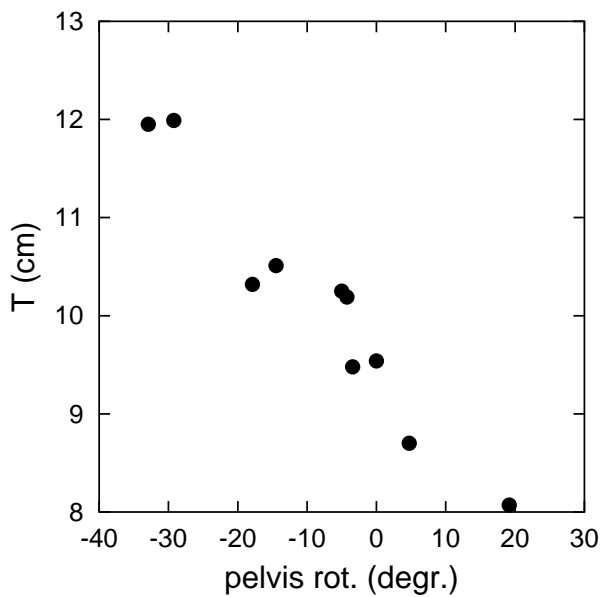

(b)

Figure 6: Figure (a) shows $T_{0}$ versus stature. Figure (b) shows $T\left(\alpha_{p}\right)$ for one of the subjects.

$T_{0}$. Multiple regression of $T_{0}$ was done for only gender and for gender and stature, resulting in $T_{0}=13.6-2.51 \times$ gender and $T_{0}=4.59-3.33 \times$ gender $+0.053 \times$ stature. If only gender is included as the independent variable, the average difference of $T_{0}$ for female ( $(9)$ and male $\left(\mathrm{O}^{7}\right)$ subjects is $2.5 \mathrm{~cm}$ (table 2). The inclusion of stature increases the coefficient of correlation slightly; for one centimeter increase of stature, $T_{0}$ tends to increase by $0.5 \mathrm{~mm}$, but partial correlation is low, see figure $6 \mathrm{a}$.

Since the ischial tuberosities converge in the sagittal direction the distance $T$ between the lower projections of the tubers must decrease with increasing pelvis angle $\left(\beta_{T}^{\alpha_{p}}<0\right)$. Figure $6 \mathrm{~b}$ shows an example where $T$ decreases from $12 \mathrm{~cm}$ to $8 \mathrm{~cm}$ for a pelvis rotation from $-34^{\circ}$ to $+20^{\circ}$. The average values of $\beta_{T}^{\alpha_{p}}<0$ for the mirror box and the pressure distribution measuring devices are $-0.054 \mathrm{~cm} /$ degree and $-0.038 \mathrm{~cm} /$ degree, see table 2 . 
Table 3: Statistical descriptives of the variables concerning the size and the shape of the contact area. Length unit is $\mathrm{cm}$.

\begin{tabular}{|c|c|c|c|c|c|c|c|}
\hline variable & $\min$ & $\max$ & $\bar{x}$ & $s_{\bar{x}}$ & $s$ & $V$ & skew \\
\hline \multicolumn{8}{|c|}{ Magnitude of the contact area in the ref. position, and the dependency on the pelvis rotation } \\
\hline$(\mathrm{cm} 2)$ & 501 & 954 & 702 & 25 & 104 & 0.15 & 0.4 \\
\hline$\beta_{A}^{\alpha_{p}} \quad(\mathrm{~cm} 2 / \mathrm{degr})$ & -6.1 & 1.0 & -2.6 & 0.4 & 1.8 & 0.7 & 0.14 \\
\hline \multicolumn{8}{|c|}{ total depth in the ref. position, and the dependency on the pelvis rotation } \\
\hline$y_{t d}^{0} \quad(\mathrm{~cm})$ & 23.8 & 29.2 & 26.6 & 0.4 & 1.7 & 0.06 & -0.4 \\
\hline$(\mathrm{cm} / \operatorname{degr})$ & -0.17 & -0.094 & -0.146 & 0.005 & 0.022 & 0.15 & 0.97 \\
\hline \multicolumn{8}{|c|}{ front depth in the ref. position, and the dependency on the pelvis rotation } \\
\hline$y_{f d}^{0} \quad(\mathrm{~cm})$ & 12.4 & 21.6 & 17.7 & 0.5 & 2.2 & 0.12 & -0.79 \\
\hline$(\mathrm{cm} / \mathrm{degr})$ & -0.065 & 0.030 & -0.009 & 0.005 & 0.023 & 2.6 & -0.70 \\
\hline \multicolumn{8}{|c|}{ back depth in the ref. position, and the dependency on the pelvis rotation } \\
\hline$y_{b d}^{0} \quad(\mathrm{~cm})$ & 6.9 & 10.0 & 8.2 & 0.2 & 0.8 & 0.10 & 0.30 \\
\hline$(\mathrm{cm} / \mathrm{degr})$ & -0.214 & -0.079 & -0.145 & 0.008 & 0.04 & 0.23 & 0.002 \\
\hline \multicolumn{8}{|c|}{ maximum width in the ref. position, and the dependency on the pelvis rotation } \\
\hline$x_{t}^{0} \quad(\mathrm{~cm})$ & 26.6 & 37.5 & 32.4 & 0.5 & 2.3 & 0.07 & -0.33 \\
\hline$(\mathrm{cm} / \mathrm{degr})$ & -0.13 & 0.09 & 0.00 & 0.01 & 0.04 & - & -0.9 \\
\hline
\end{tabular}

Table 4: Results of stepwise linear regression of the variables concerning the size and the shape of the contact area. The results for $y_{t d}^{0}, \beta_{t d}^{\alpha_{p}}, x_{t}^{0}$, and $\beta_{m w}^{\alpha_{p}}$ have been excluded from this table because no significant correlation was found. Units are centimetres and degrees.

\begin{tabular}{lrrrrrrrr}
\hline & & const & $\Delta$ sips & $\Delta$ sias & $s s_{\text {avg }}$ & $\%$ fat & ecto & mult. r \\
\cline { 3 - 7 }$A_{0}$ & $\left(\mathrm{~cm}^{2}\right)$ & 51.3 & 44.3 & & & 10.2 & 0.81 \\
$\beta_{A}^{\alpha_{p}}$ & $\left(\mathrm{~cm}^{2} / \mathrm{degr}\right)$ & 1.32 & & & & -0.21 & & 0.77 \\
$y_{b}{ }^{0}$ & $(\mathrm{~cm})$ & 9.29 & & & & & -0.34 & 0.55 \\
$\beta_{y_{b}}^{\alpha_{p}}$ & $(\mathrm{~cm} / \mathrm{degr})$ & -0.29 & & -0.014 & 0.028 & & & 0.74 \\
$x_{t}^{0}$ & $(\mathrm{~cm})$ & 15.9 & 1.19 & & & 0.23 & & 0.83 \\
\hline
\end{tabular}

Contact area The results for the size $\left(A_{0}\right)$ and the shape of the contact area are given in table 3. The shape is represented by the front depth, $y_{f d}$, the back depth, $y_{b d}$, and the total 
The shape of the contact area and the shange of the size

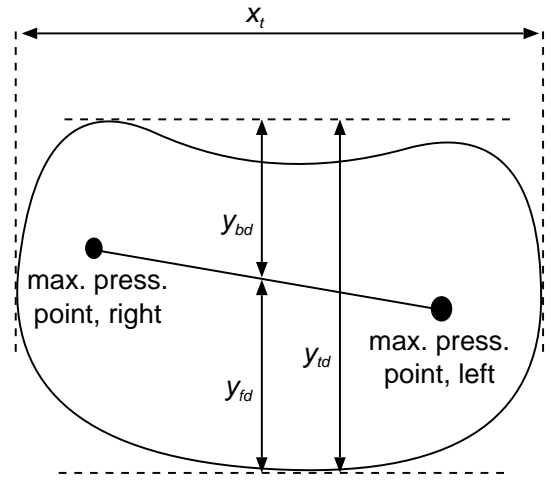

(a)

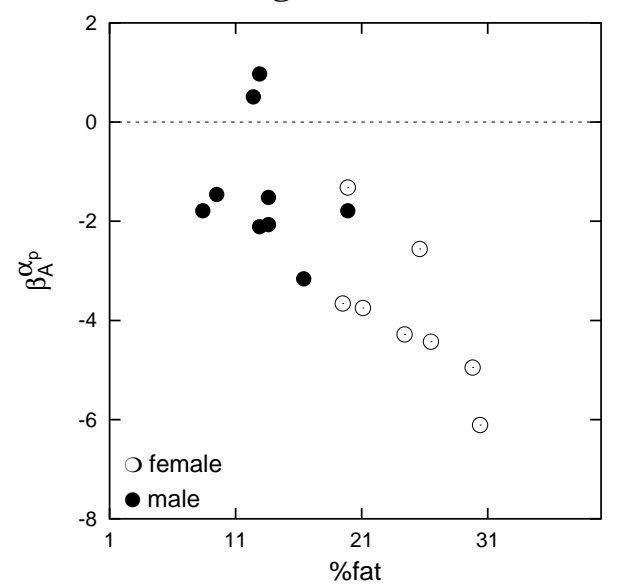

(b)

Figure 7: Figure (a): the front depth, $y_{f d}$, the back depth, $y_{b d}$, and the total depth, $y_{t d}$, and width, $x_{t}$, of the contact area. Figure (b): $\beta_{A}^{\alpha_{p}}\left(\mathrm{~cm}^{2} / \mathrm{degr}\right)$ as a function of $\%$ fat.

depth, $y_{t d}$, see figure 7(a). Multiple regression (stepwise; level of significance for the Tstatistic was 0.05 ) was applied for the measured body characteristics, see table 4 . The size of the contact area $A_{0}=702(104) \mathrm{cm}^{2}$. The main explaining quantity for the variance of $A_{0}$ and $x_{t}$ is the percentage of subcutaneous fat (\% fat). The change of the size of the contact area with the pelvis angle $\left(\beta_{A}^{\alpha_{p}}\right)$ is, apart from two subjects, negative (table 4 and figure $7 \mathrm{~b}$ ). This indicates that forward bending (increasing $\alpha_{p}$ ) reduces the size of the contact area. A significant relationship was only found for \%fat. Thus lean and relatively thin, tall subjects show lower decrease of $A$ than subjects that are more endomorphic for the same variation of the pelvis rotation.

Sitting force and average pressure The tables 5 and 6 show the statistical descriptives of the sitting force and the results of the multiple regression with body characteristics. The sitting force in the reference posture $F_{s}=508(79) \mathrm{N}$, and depends on body mass ( $r=$ 
Table 5: Statistical descriptives of the variables related to the sitting force, its sagittal position and the average pressure.

\begin{tabular}{lllllllll}
\hline variable & $\min$ & $\max$ & $s_{\bar{x}}$ & $s$ & $V$ & skew \\
\hline
\end{tabular}

Sitting force in the ref. position, and the dependency on the pelvis rotation

$\begin{array}{lllllllll}F_{s} & (\mathrm{~N}) & 303 & 647 & 508 & 18 & 79 & 0.16 & -0.72\end{array}$

$\begin{array}{lllllllll}\beta_{F_{s}}^{\alpha_{p}} & \text { (N/degr) } & -0.92 & 0.47 & -0.22 & 0.09 & 0.37 & 1.72 & -0.30\end{array}$

Sitting force related to body weight in the ref. position
$F_{s} / F_{G}$
0.60
0.85
$0.78 \quad 0.013$
$\begin{array}{lll}0.06 & 0.07 & -1.67\end{array}$

Sagittal location of the line of action of the sitting force in the ref. position, and the dependency on the pelvis rotation

$\begin{array}{lrrrrrrrr}y_{F_{s}} & (\mathrm{~cm}) & 8.5 & 16.9 & 13.8 & 0.5 & 2.2 & 0.22 & -0.70 \\ \beta_{y_{F_{s}}}^{\alpha_{p}} & (\mathrm{~cm} / \text { degr }) & 0.09 & 0.16 & 0.12 & 0.01 & 0.03 & 0.20 & 0.15\end{array}$

Average pressure in the ref. position, and the dependency on the pelvis rotation

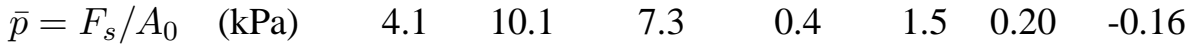
$\begin{array}{lllllllll}\beta_{\bar{p}}^{\alpha_{p}} & (\mathrm{kPa} / \mathrm{degr}) & -0.016 & 0.040 & 0.02 & 0.00 & 0.02 & 1.00 & -1.06\end{array}$

0.91). A significant relationship between $F_{s}$ and $\alpha_{p}$ was not found. $\beta_{F_{s}}^{\alpha_{p}}$ Shows a tendency to negative values (see also figure 8) which means that for most subjects $F_{s}$ decreases when the pelvis is rotated in forward direction. Multiple regression did not give significant explaining variables. The average fraction $F_{s} / F_{G}$ ( $F_{G}=$ body weight), transmitted via the seat, is 0.78 with only small deviations $(V=7 \%)$, not explained by body characteristics.

The sagittal coordinate, $y_{F_{s}}$, of the line of action of the sitting force was only marginally $(r=0.59)$ explained by the sagittal dimension of the pelvis, $s s_{\text {avg }}$. The regression coefficient $(-1.98 \mathrm{~cm} / \mathrm{cm})$ shows that an increase of the depth, $s s_{\text {avg }}$, of the pelvis of $1 \mathrm{~cm}$ corresponds with a displacement of about $-2 \mathrm{~cm}$ of $y_{F_{s}}$. The values of $\beta_{y_{F_{s}}}^{\alpha_{p}}$ are definitely positive, which means that a forward rotation causes a forward translation of $y_{F_{s}}{ }^{3}$.

\footnotetext{
${ }^{3}$ This translation may not be confused with the movement of the points of maximum pressure, which shows varying relationship with $\alpha_{p}$.
} 
Table 6: Results of stepwise linear regression of the variables, concerning the sitting force and the average pressure, with the body characteristics (mass in $\mathrm{kg}$ and $s s_{\text {avg }}$ in $\mathrm{cm}$ ).

\begin{tabular}{lrrrrrrr}
\hline & & const & mass & ssavg & gender & endo & mult. r \\
\cline { 3 - 7 }$F_{s}$ & $(\mathrm{~N})$ & -25.5 & 7.98 & & & & 0.91 \\
$y_{F_{s}}$ & $(\mathrm{~cm})$ & 47.5 & & -1.98 & & & 0.59 \\
$\beta_{y}^{\alpha_{p}}$ & $(\mathrm{~cm} / \mathrm{degr})$ & 0.47 & -0.020 & & & 0.55 \\
$\bar{p}$ & $(\mathrm{kPa})$ & 6.3 & & & 2.1 & & 0.75 \\
$\beta_{\bar{p}}^{\alpha_{p}}$ & $(\mathrm{kPa} /$ degr $)$ & $-5.8 \mathrm{e}-03$ & & & & $9.1 \mathrm{e}-03$ & 0.65 \\
\hline
\end{tabular}

In the reference posture the overall (all subjects) average pressure is $\bar{p}=7.3 \mathrm{kPa}$ (which exceeds the limit for decubitus, $\bar{p}=7 \mathrm{kPa}$, defined by Hobson (1988)). $F_{s}$ is correlated with body mass. Since for men $F_{G}$ is higher than for women, the same is valid for $F_{s}$. The larger size of the contact area for women and the smaller sitting force do account for the lower average pressure for women. It depends significantly on gender, with regression coefficient $2.1 \mathrm{kPa}$, which expresses that the average pressure shows a systematic difference for men and women. For women it is lower as a result of increased size of the contact area.

The average pressure increases with forward rotation, $\beta_{\bar{p}}^{\alpha_{p}}>0$, despite the decrease of $F_{s}$. Apparently the decrease of $A_{0}$ compensates for the decrease of $F_{s}$. It is mainly correlated with the endomorphic index. Thus, for highly endomorphic persons the average pressure increases more rapidly with forward rotation of the pelvis.

Pressure distribution descriptors Tables 7 and 8 summarise the results of the parameters that describe the pressure distribution and the results of the regression analysis. It turned out that the ectomorphic index, and in one case stature, were the only explaining variables. 
The dependency of the sitting force on the angle of pelvis rotation.

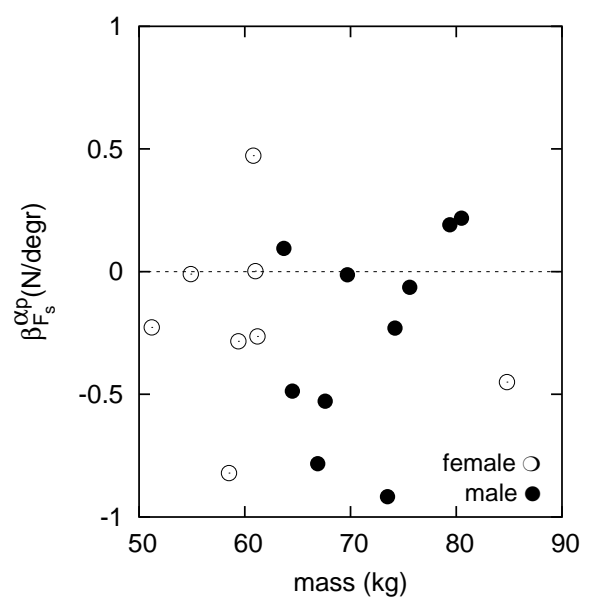

Figure 8: The value of $\beta_{F_{s}}^{\alpha_{p}}$ is mainly negative.

Maximum pressure In the reference posture the maximum pressure, $p_{m}=173(93) \mathrm{kPa}$ for the left and 176(91) $\mathrm{kPa}$ for the right side. Multiple regression shows a significant relationship with ectomorphy.

The pressure distribution depends on the pelvis angle $\alpha_{p}$. Figure 9 shows the average of the left and the right maximum pressure as a function of the pelvis angle for one of the subjects. Usually $p_{m}\left(\alpha_{p}\right)$ shows a maximum for the reference posture $\left(\alpha_{p}=0\right)$. To describe the relationship and to characterize it by a small number of parameters, for each subject $p_{m}$ was fitted to an exponential function: $\widehat{p}_{m}^{\alpha_{p}}=c \cdot e^{-\frac{\left(\alpha_{p}-b\right)^{2}}{a^{2}}}$, where $c$ corresponds with the maximum pressure in the reference posture, $b$ with the reference angle of the pelvis rotation, and $a$ with the width of the graph. The width of the function equals the distance between the inflection points: $\alpha_{p(1,2)}=b \pm \frac{1}{2} a \sqrt{2}$. The width $w$ of the function is $w=a \sqrt{2}$.

A high value of $w$ indicates a relatively slow decrease of the maximum pressure for a deviation from the reference posture. If $w$ is small, then the maximum pressure decreases 


\section{The maximum pressure}

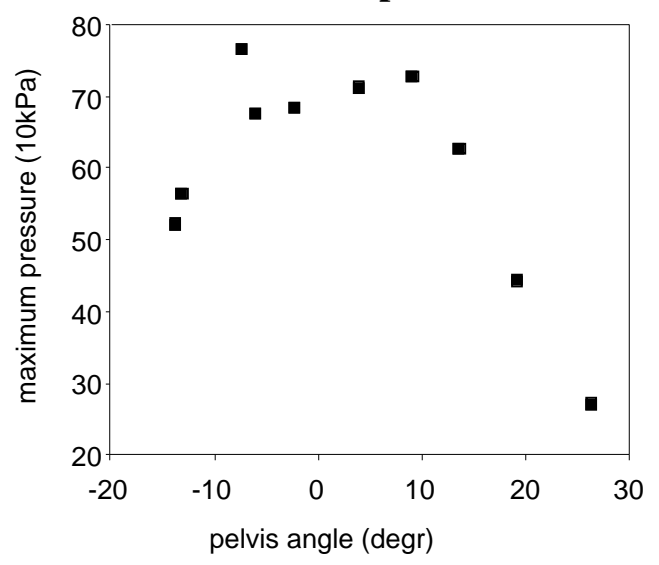

Figure 9: Average of the left and right maximum pressure values as a function of the pelvis angle $\alpha$ for one of the subjects.

rapidly with $\alpha_{p}$. The value of $w$ was $37(12)^{\circ}$ with maximum and the minimum values $20^{\circ}$ and $62^{\circ}$, see table 7. Multiple regression showed no significant dependencies.

If the graph of the function is steep, this means that $p_{m}$ changes rapidly with $\alpha_{p}$. Therefore the rate of change of $p_{m}$ with $\alpha_{p}$ can be represented by the slope at the inflection points: $\left(\beta_{p_{m}}^{\alpha_{p}}\right)_{\alpha_{1,2}}=\frac{\partial p_{m}\left(\alpha_{1,2}\right)}{\partial \alpha_{p}}= \pm c \sqrt{2} \times e^{\frac{1}{2} \sqrt{2}}$. The computations showed that $\beta_{p_{m}}^{\alpha_{p}}=52(25) \mathrm{N} /\left(\mathrm{cm}^{2}\right.$ degr $)$. Linear regression yields: $\left(\beta_{p_{m}}^{\alpha_{p}}\right)_{\alpha_{1,2}}=11.5+13.1 \times$ ecto with $r=0.70$. The ectomorphic index, ecto, is the only explaining variable.

Pressure gradient The pressure gradient has been approached by two methods, which have been explained in the appendix. The circular gradient, $G^{C}$ represents the changes of the pressure by a single number. The transverse gradient discerns between the medial and the lateral components. Both gradients have been computed for the left and the right parts.

Theoretically, at a maximum pressure point $(r=0)$ the pressure gradient $G^{\prime}=0$. For increasing distance, $r$, the gradient first increases until a maximum, then decreases. 
Table 7: The statistical descriptives of the maximum pressure $(\mathrm{kPa})$ and the pressure gradient $\left(10^{3} \mathrm{kPa} / \mathrm{m}\right)$.

\begin{tabular}{llllllll}
\hline variable & $\min$ & $\max$ & $\bar{x}$ & $s_{\bar{x}}$ & $s$ & $V$ & skew
\end{tabular}

Left, right and average maximum pressure in the ref. position

$\begin{array}{lllllllll}p_{m, l} & (\mathrm{kPa}) & 56 & 350 & 173 & 23 & 93 & 0.54 & 0.50 \\ p_{m, r} & (\mathrm{kPa}) & 85 & 350 & 176 & 23 & 91 & 0.52 & 0.79 \\ p_{m} & (\mathrm{kPa}) & 71 & 350 & 170 & 19 & 78 & 0.46 & 0.60\end{array}$

Computed coefficients of the fitting equation

$\begin{array}{lrrrrrrrr}c & (\mathrm{kPa}) & 75 & 342 & 182 & 22 & 87 & 0.48 & 0.44 \\ w(=a \sqrt{2}) & (\mathrm{degr}) & 19.6 & 61.7 & 36.7 & 3.0 & 11.8 & 0.32 & 0.86\end{array}$

The dependency of the maximum pressure on the pelvis rotation $\begin{array}{lllllllll}\left(\beta_{p_{m}}^{\alpha_{p}}\right)_{\alpha_{1,2}} & (\mathrm{kPa} / \mathrm{degr}) & 215 & 980 & 521 & 63 & 250 & 0.48 & 0.44\end{array}$

Circular and lateral (left and right) pressure gradients in the ref. position

$\begin{array}{lcccccccc}G_{0}^{C} & (\mathrm{kPa} / \mathrm{m}) & 1.48 & 5.44 & 3.1 & 0.33 & 1.27 & 0.41 & 0.24 \\ G_{0}^{T} & (\mathrm{kPa} / \mathrm{m}) & 2.27 & 16.8 & 6.9 & 1.1 & 4.2 & 0.60 & 1.17 \\ G_{l, 0}^{T} & (\mathrm{kPa} / \mathrm{m}) & 2.10 & 16.6 & 6.6 & 1.1 & 4.1 & 0.63 & 1.20 \\ G_{m, 0}^{T} & (\mathrm{kPa} / \mathrm{m}) & 2.44 & 16.9 & 7.3 & 1.1 & 4.2 & 0.58 & 1.12 \\ G_{l, 0}^{T} / G_{m, 0}^{T} & (\mathrm{kPa} / \mathrm{m}) & 0.70 & 1.06 & 0.89 & 0.03 & 0.11 & 0.12 & 0.30\end{array}$

This means that the region of maximum pressure gradient (maximum drainage of fluid) is not located at the point of maximum pressure, but in a region around this point. Since a computed point of maximum pressure does probably not coincide with the centre of a measuring element, in practice $G^{\prime}(r=0) \neq 0$. Figure 10 shows the circular pressure gradient for one of the subjects (computed with eq. 2, appendix).

The circular gradient in the reference posture is $G^{C}=3.14(1.33) \mathrm{kPa} / \mathrm{m}$. Multiple regression yields only ectomorphy and stature as explaining variables. Since the circular gradient is a single, overall measure for the pressure gradient, not showing directional dependency, the lateral and the medial components of the transverse gradient have been 
Table 8: Coefficients of the regression results of the pressure and the pressure gradient parameters (stature in $\mathrm{m}$ ).

\begin{tabular}{lrrrrc}
\hline & & const & ecto & stature & mult. $\mathrm{r}$ \\
\cline { 3 - 6 }$p_{m, l}$ & $(\mathrm{kPa})$ & 63.9 & 36.5 & & 0.53 \\
$p_{m, r}$ & $(\mathrm{kPa})$ & 2.7 & 54.1 & & 0.77 \\
$p_{m}$ & $(\mathrm{kPa})$ & 38.6 & 42.2 & & 0.73 \\
$c$ & $(\mathrm{kPa})$ & 40.0 & 45.6 & & 0.70 \\
$\left(\beta_{p_{m}}^{\alpha_{p}}\right)_{\alpha_{1,2}}$ & $(\mathrm{kPa} / \mathrm{degr})$ & 115 & 131 & & 0.70 \\
$G_{0}^{C}$ & $(\mathrm{kPa} / \mathrm{m})$ & -6.25 & 0.56 & 0.044 & 0.90 \\
$G_{0}^{T}$ & $(\mathrm{kPa} / \mathrm{m})$ & -1.03 & 2.55 & & 0.80 \\
$G_{l, 0}^{T}$ & $(\mathrm{kPa} / \mathrm{m})$ & -1.25 & 2.51 & & 0.80 \\
$\left(G_{m, 0}^{T}\right.$ & $(\mathrm{kPa} / \mathrm{m})$ & -0.81 & 2.59 & & 0.80 \\
\hline & & & & &
\end{tabular}

Table 9: Overview of the main predicting variables for the pressure distribution parameters.

\begin{tabular}{lcccccc}
\hline variable & body mass & gender & stature & ecto & $\%$ fat & $\alpha_{p}$ \\
\cline { 2 - 6 }$T$ & & $\mathrm{X}$ & $\mathrm{X}$ & & & $\mathrm{X}$ \\
$A$ & $\mathrm{X}$ & & & & $\mathrm{X}$ & $\mathrm{X}$ \\
$F_{S}$ & $\mathrm{X}$ & & & & & \\
$\bar{p}$ & & $\mathrm{X}$ & & & $\mathrm{X}$ \\
$p_{m}$ & & & & $\mathrm{X}$ & $\mathrm{X}$ \\
$G$ & & & & $\mathrm{X}$ & $\mathrm{X}$ \\
\hline
\end{tabular}

computed (eqs. 3 and 4, appendix). Linear regression gives $G_{l}^{T}=-0.43+0.964 \times G_{m}^{T}$ with coefficient of correlation $\rho=0.98$. The negative intercept $(-0.43)$ shows that the lateral gradient is slightly smaller than the medial gradient. Ectomorphy is practically the only significant explaining variable of the transverse gradient. Linear regression of the average (lateral, medial, left, right) transverse gradient and the average circular gradient, both in the reference posture, gives $G_{0}^{C}=1.18+0.28 \times G_{0}^{T} ; r=0.93$. The circular pressure gradient is significantly smaller than the lateral gradient. 


\section{Computed circular gradient}

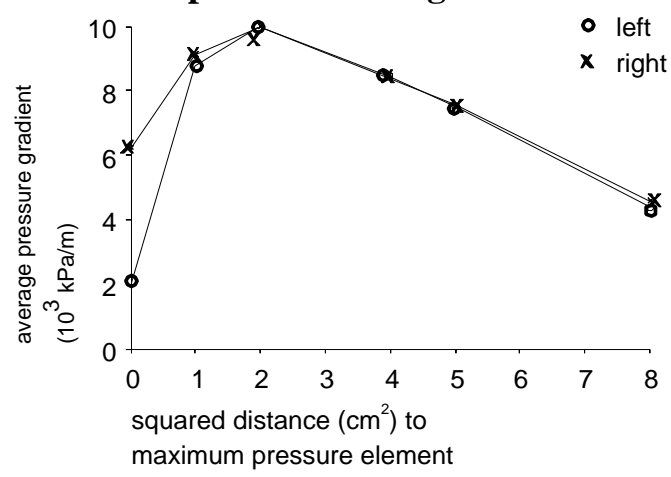

Figure 10: Example of the computed circular gradient as a function of $r^{2}$.

Overview of the predicting variables Table 9 gives an overview of the main predicting variables for the investigated parameters of the pressure distribution.

\section{Discussion}

Generalisation Since our subjects were healthy students of a limited range of age, a generalization of the results, such as the application to children or the elderly, needs additional measurements.

Maximum pressure and pressure gradient The maximum pressure, that was found for the reference posture, is $170(19) \mathrm{kPa}$. Results from reported research using a comparable measurement setup, flat/basic supported sitting, showed lower values (Houle, 1969), or comparable values (Minns et al., 1984). The reasons are not difficult to understand. Firstly, in our research the subjects took the upright posture, and stretched their back. Secondly they were requested to rotate the pelvis until they experienced the maximum load under the seating bones. In this respect the results compare to the results of Minns et al. (1984). 
Stumbaum (1983) and Brienza and Karg (1998) measured on flat surfaces, but since they used a backrest, they found lower maximum pressure values, 40-180 kPa.

The same reasoning can be done for the pressure gradient. In this research the circular gradient and the transverse gradient were $3.1(1.27) 10^{3} \mathrm{kPa} / \mathrm{m}$ and $6.9(4.2) 10^{3} \mathrm{kPa} / \mathrm{m}$. The lower values found by Hobson (1988) and Brienza and Karg (1998) can be attributed to using a soft surface (cushion) and to reshaping the surface. Although Bader and Hawken (1986) used cushions and multiple support, their results are in the same order as those in this paper. The reasons for this are not clear.

The influence of body characteristics on the maximum pressure are contrary to the results of Garber and Krouskop (1982), who found a significant influence of the body type (thin, average, obese) on the location of the maximum pressure (bony region or soft tissue region), but the value of the maximum pressure was only weakly influenced by body type. We found a significant influence of somatotype on the maximum pressure, and a significant, but smaller contribution of the body anthropometry (stature) on the pressure gradient. A change of the maximum pressure as a result of a rotation of the pelvis was found to be maximal for high ectomorphy. This means that a reduction of the maximum pressure by rotating the pelvis is most effective for a typically ectomorph person. This could mean that the transmission of the sitting force is taken over by the hamstring muscles or the gluteal muscles at smaller deviations from the reference posture than for low ectomorphic index (more adipose tissue).

Contact area The size of the contact area, $702 \mathrm{~cm}^{2}$ with standard deviation $104 \mathrm{~cm}^{2}$, is of the same order of magnitude as found by Aissaoui et al. (2001). It depends mainly on \%fat mainly, and the distance between the SIPSes has a small, but significant contribution. 
This means that females tend to have a larger contact area than males. The same is valid for a change of the contact area if the pelvis is rotated; for ectomorph people the contact area changes less quickly than people having more subcutaneous fat.

Average pressure The average pressure in the reference posture, $7.3 \mathrm{kPa}$ and standard deviation $1.5 \mathrm{kPa}$, is in the same order as found by Bader and Hawken (1986), despite the fact that they used a cushion as well as multiple support. Possibly the contact area was smaller in that research. The main explaining quantity is gender: for males the average pressure exceeds that of females by about $2.1 \mathrm{kPa}$. This tendency can be explained easily since (i) the contact area of males is smaller (less \%fat) and (ii) their body weight is larger, see table 4 . However, the dependency of the average pressure on a rotation of the pelvis has a positive correlation with the endomorphic index, see table 6 . This means that typically 'thin' people show less variation of the average pressure for a rotation of the pelvis than less thin people.

Sitting force The sitting force is slightly higher than the force found by Aissaoui et al. (2001). A larger difference was expected, since using a backrest induces easily a decrease of one third of the sitting force (Souther et al., 1974). Since in our research only the pelvis was rotated and not the upper body the centre of mass shows no substantial variation. Therefore it is not surprising that the sitting force depends only on the body mass and not on the rotation of the pelvis. The force reduction resulting from a rotation of the trunk, and thus from a shift of the centre of mass, can easily be explained with a simple biomechanical model, and was confirmed experimentally by Henderson et al. (1994). 
Distance between maximum pressure points The distance between the maximum pressure points was derived from the locations of these points. Locating the maximum pressure points using the mirror box device can be improved by using an enhanced white rubber foil resulting in better sensitivity in the brightest regions. The computation using the contourline method was done using a specific set of contour parameters (appendix), including among other things a degree of smoothing. In this research a reasonable setup was selected. It is not expected that further optimization of these parameters will give significant improvement. The correlation between the contourline data and the mirror box data is sufficiently strong to accept the current level of accuracy, compare with table 2.

The distance between the points of maximum pressure, $12.3 \mathrm{~cm}$, and standard deviation $1.5 \mathrm{~cm}$, agrees with the results of Stumbaum (1983). Smaller distances were found by Houle (1969), but in that report the measurement setup to obtain this measure was not described, and by van Engelen (1988), who measured the distance between the deepest impressions on bicycle saddles. In practice this distance is defined variously, depending on the context (gynaecology, forensic determination of gender, sitting comfort, etc) so that a comparison is not always straightforward. For instance, the distance between the inner aspects of the ischial tuberosities (the transverse diameter of the inferior aperture of the pelvis also called the bituberous diameter) is about $85 \mathrm{~mm}$ for males and $118 \mathrm{~mm}$ for females (Williams et al., 1989). The change of the distance for a $10^{\circ}$ forward rotation of the pelvis was found $-4 \mathrm{~mm}$ to $-5 \mathrm{~mm}$.

The movement of the points of maximum pressure was investigated for quasi-static lateral pelvis rotation. If different ways of sitting are investigated, consideration of dynamics and types of motion must be included. For instance, on a bicycle saddle the points of maximum pressure show a circular motion in lateral direction (Moes, unpublished data). 
Rotation of the pelvis The helical axis (see appendix) for the current type of rotation and translation, is located above the sitting plane. It shows a large uncertainty, mainly as a result of the varying amount of sliding of the ischii over the inner aspect of the skin. In the measurement setup of Stumbaum (1983) it was located below the trunk, but in that research only a rotation of the backrest was considered, while no attention was payed to a rotation of the pelvis. Moreover, in that research this location was considered important to prevent sliding forces along the back during rotation of the back rest (Hemdauszieheffekt). In his thesis he did not consider the possibility of the seating bones sliding over the inner aspect of the skin.

Differences within subjects and among subjects for the maximum backward and forward rotation depend not only on physiological and anatomical factors; the stiffness characteristics of the spine and its ligaments are possible additional contributors to such asymmetry.

Model ischial tuberosities The radius of the model for the ischial tuberosities could not be computed since the ischial tuberosities showed an unpredictable sliding behaviour over the inner aspect of the skin. The exact reasons for the varying sliding behaviour can only be guessed. For sure, varying muscle and ligament characteristics, such as stiffness, trainedness and muscular pretension could play a significant role, but other factors, e.g. psychophysiological, should be considered in addition.

\section{Conclusions}

The pressure distribution has been described by the maximum pressure, the pressure gradient, the average pressure, and the location of the points of maximum pressure for subjects 
sitting on a flat, hard and horizontal support. The variation of the posture was defined by a rotation of the pelvis. Using multiple regression techniques the relationships between these quantities and (i) a set of body characteristics, and (ii) the pelvis rotation were computed. Taking into consideration the boundary conditions of the research it can be concluded that the pressure distribution for a specific angle of lateral rotation of the pelvis can be predicted by ectomorphic index, mass, gender, \%fat and stature. It was shown that the maximum pressure depends on the angle of pelvis rotation. This influence is stronger for increasing ectomorphy.

The radius of the curvature of the ischium model (eq. 1, appendix) can not be derived from the measurements of the location of the maximum pressure points, because the ischial tuberosities show an unpredictable sliding over the inner aspect of the skin while the subject rotates the pelvis. Therefore the angle between the ischial tuberosities and the curvature can not separately be determined from these data.

The distance between the points of maximum pressure is predicted by gender, stature, and the angle of the pelvis rotation. For each $10^{\circ}$ of rotation, the distance is changed by about $4 \mathrm{~mm}$. As was expected it decreases for forward rotation and increases for backward rotation. The front depth can not be predicted since it is still unknown what conditions cause sliding or rolling of the ischial tuberosity over the inner aspect of the skin. The values of the distance between the ischial tuberosities are not consistent with those found in literature since the measurement setup was different.

The maximum forward or backward rotation of the pelvis showed no relationship with gender or other body characteristics. 


\section{Acknowledgements}

Special thanks go to Henk Lok, who constructed the antenna, the various versions of the pressure distribution measuring device, the mirror box, including the corrugated white foil. Hans Houtkamp had a substantial contribution to the calibration of the measuring devices and the measurement setup.

\section{Appendix}

Location of the points of maximum pressure To obtain the outer contact contour, the size of the contact area, and the location of the maximum pressure points, the mirror box images were digitized and analyzed with an image analysis program (Fox and Ulrich, 1995). Because of the resolution of the brightness of the images the brightest areas do not show as small spots, but as an area around the actual, but unknown point of maximum pressure. The (unweighted) centre of mass-routine (CM-binary), that locates the $X$ and $Y$ coordinates of the geometric centre of mass for the pixels of the image according to $C M \operatorname{Binary}(X)=$ $(1 / N) \sum_{i=1}^{N} X_{i}$ and $C M \operatorname{Binary}(Y)=(1 / N) \sum_{i=1}^{N} Y_{i}$ where $N$ is the number of pixels in the image, was applied to locate the point of maximum pressure for the contour of the area of maximum optical density.

Sitting force The sitting force was obtained by summing the four vertical force components of the Kistler force plate. The point of action of the force was computed from the ratios of these components. The conversion of the output values of the pressure distribution measuring device to the pressure values is discussed in Moes (1999). The locations of the maximum pressure points were also obtained from the pressure distribution data. The geo- 
metric centre of the highest pressure contourline (left and right) of the pressure distribution was considered the 'point' of maximum pressure, see figure 3.

Sagittal location The sagittal location, $y_{f}$, of the points of maximum pressure is the distance to the front edge of the 'seat'. It is computed as the average of the left and the right locations: $y_{f}=0.5\left(y_{f_{l}}+y_{f_{r}}\right)$. The value of $y_{f}$ depends on the distance between the front edge and the backboard, but only its variation was used to obtain the relationship with the angle of the pelvis rotation: $\beta_{y_{f}}^{\alpha_{p}}=d y_{f} / d \alpha_{p}$

Distance between the points of maximum pressure The assumption was made that the location of the points of maximum pressure has a direct relationship with the lower aspects of the ischial tuberosities. Since the ischial tuberosities have an elongated shape and converge in the sagittal direction the distance between the points of maximum pressure depends on the angle of the pelvis rotation. If we know the curvature of the ischial tuberosities and the angle between the bones, this distance can be computed as a function of the rotation of the pelvis. Therefore the lower part of the shape of the ischial tuberosities was modelled as a section of a circular disk with radius $r$, see figure 11. Since the ischial tuberosities converge in forward direction $T$ decreases with forward rotation. The angle between the left and the right section is $\gamma$. Points $A$ and $B$ are the lower aspects of the right and left discs. If the discs roll over an angle $\Delta \alpha_{p}$ their lower aspects move from $A$ to $A^{\prime}$ and from $B$ to $B^{\prime}$ so that $\Delta s=r \times \Delta \alpha_{p}$. Then:

$$
\beta_{T}^{\alpha_{p}}=\frac{d T}{d \alpha_{p}}=-\frac{2 r \times d \alpha_{p} \times \sin (\gamma / 2)}{d \alpha_{p}}=-2 r \sin (\gamma / 2)
$$

so that $\beta_{T}^{\alpha_{p}}$ is indeed negative.

During rotation of the pelvis the ischial tuberosities move with respect to the inner 


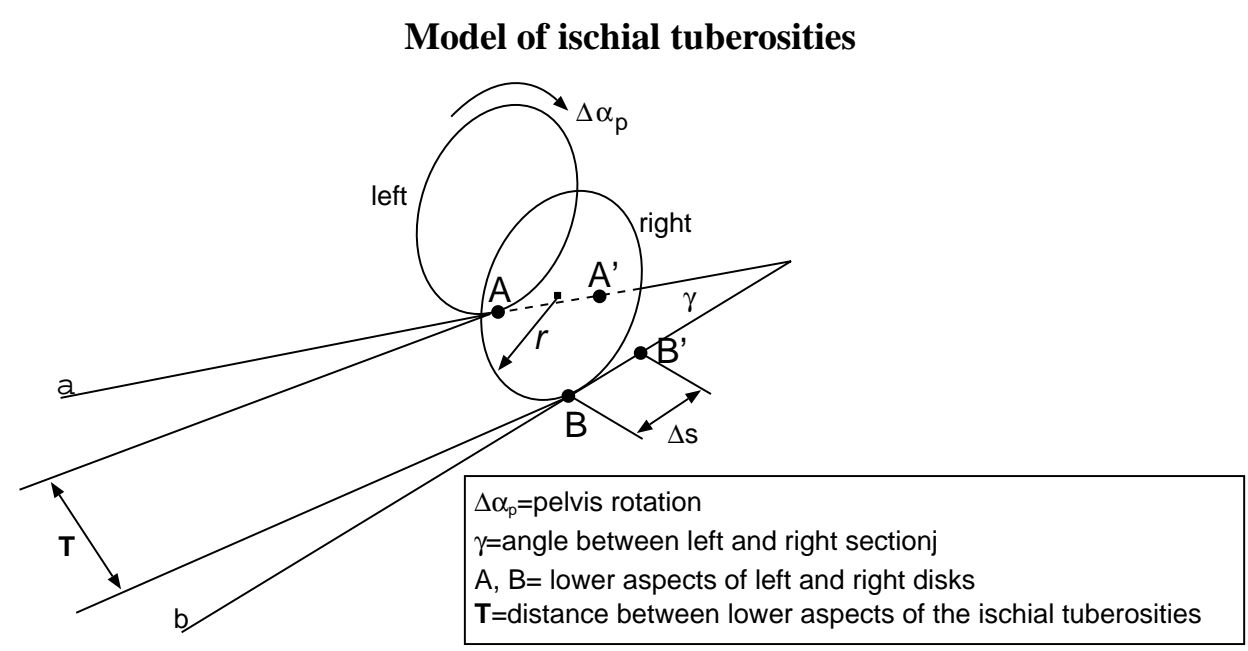

Figure 11: The circular disc model of the ischial tuberosities.

side of the skin. This movement can be rolling, sliding, or a combination of rolling and sliding. In case of only rolling the point of contact moves according to eq. 1 so that $\gamma$ can be derived from the trajectories of the maximum pressure points. If $\Delta T$ and $\Delta \alpha_{p}$ are known from measurements, the radius $r$ follows from eq. 1. But if also sliding occurs, only the product $r \sin (\gamma / 2)$ can be determined.

Whether sliding occurs depends on muscular activation involved in the movement. Such activation is not only determined by specific anatomical and biomechanical characteristics, but also by individual motorial habits. Moreover, the coefficient of friction between the ischial tuberosities and the inner surface of the skin is unknown. The occurrence of sliding is indicated by the sagittal displacement of the points of maximum pressure during rotation of the pelvis. If only rolling is involved the function $y_{f}\left(\alpha_{p}\right)$ is monotonously decreasing. The reverse is not necessarily true; a monotonously decreasing relationship is no proof for the absence of sliding. The actual relationship depends on the relative amounts of rolling and sliding. 
Helical axis of rotation The movement of the pelvis consists of a rotation and a translation. The combined movement can be expressed as a rotation around the helical axis (screw axis), which runs in lateral direction. The location of this axis was computed by considering the overall movement of the antenna. For each measurement the lateral intersection of the extrapolated antenna line with the antenna line of the reference posture was considered as the pivot point of the helical axis. If the movement of the ischial tuberosities with respect to the inner surface of the skin can be completely attributed to rolling without sliding, the helical axis of rotation is located at the interface between the ischial tuberosities and the inner surface of the supporting skin. But if also sliding occurs, the helical axis is at a different height $(z)$.

Range of pelvis rotation Since $T$ depends on $\alpha_{p}$, the maximum forward and the maximum backward rotation determine the range of $T$. These values are determined by specific anatomical entities, such as such as the flexibility of the lower spine and the stiffness and pretension of the involved muscle groups.

Pressure distribution data and image analysis The raw pressure data were converted to the actual pressure values (Moes, 1999). From these data the maximum pressure $p_{m}$ and the circular gradient were computed. The transverse gradient and the positions of the maximum pressure point were computed from the contourline plots (GPCP, G(eneral) $\mathrm{P}$ (urpose) $\mathrm{C}$ (ontourline) $\mathrm{P}($ ackage), implemented on a Convex main frame) (Batten and Walters, 1971). The main control variables were (i) grid interval=0.5, (ii) eight cells are used to compute the basic grid for the contour lines, (iii) eight cells are used to compute the 'interim gradient', (iv) refinement for smoothing is 2 . The actual algorithms are described in the manual (Batten and Walters, 1971). Figure 3 shows an example of a contourline plot 


\section{Noise reduction of the mirror images}

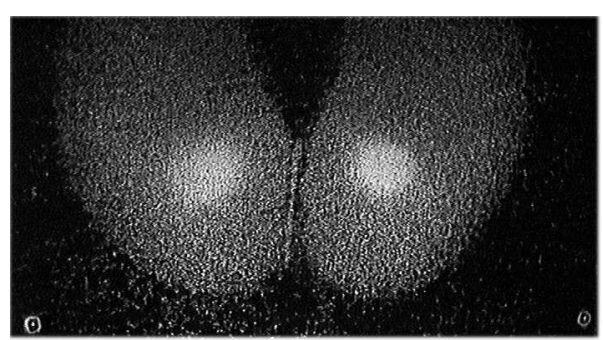

(a) Image from the mirror box.

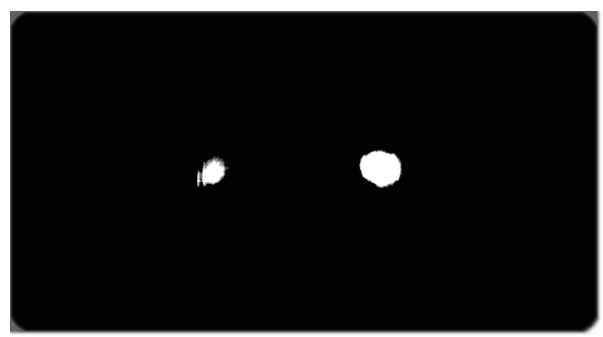

(c) Same image showing only maximum pressure areas.

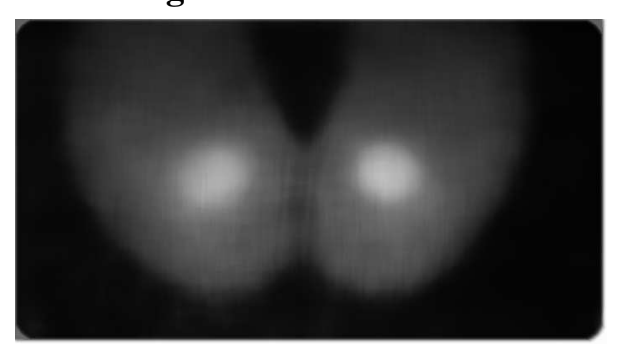

(b) Same image after application of noise reduction.

Figure 12: Three stages of image processing.

where the first bit values, not relevant for $p_{m}$ and the pressure gradient, have been omitted.

The original mirror box images (figure 12a) were processed (median method) for noise reduction (figure 12b) and increase of contrast (figure 12c). The maximum contrast images were used for the computation of the geometric centre.

Pressure gradient If the location on the support is defined by the carthesian coordinates, $x$ and $y$, and the third axis, perpendicular to the support plane, represents the pressure as a function of the location, then the resulting graph shows a 'pressure mountain', $p(x, y)$. The gradient, $G$, of the pressure is a continuous function, defined on $p(x, y): G(x, y)=$ $\sqrt{\left(\frac{\partial p}{\partial x}\right)^{2}+\left(\frac{\partial p}{\partial y}\right)^{2}}$. The measured pressure distribution is always given as a discrete image 


\section{Pressure mountain}

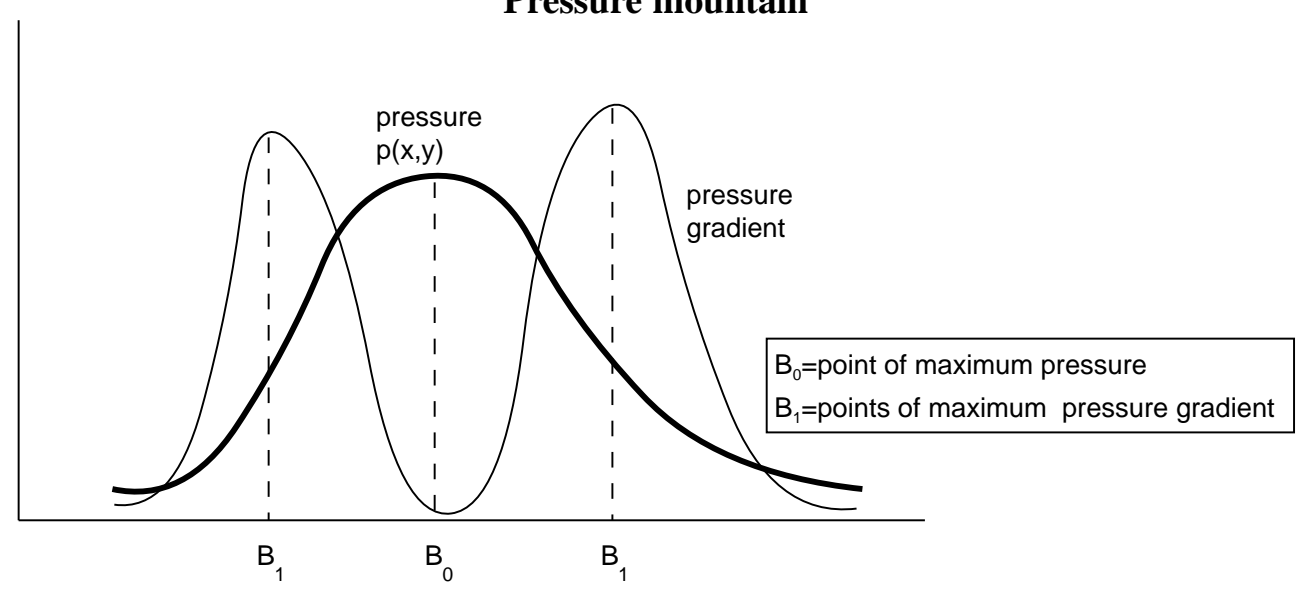

Figure 13: Two-dimensional lateral cross section of the pressure mountain $p(x, y)$.

of the actual pressure distribution. Published investigations used various distances and number of elements to compute the pressure gradient. The gradient is maximal where the $p(x, y)$ is steepest $\left(\mathrm{B}_{1}\right.$ in figure 13$)$, zero at the top $\left(\mathrm{B}_{0}\right)$, and decreases outside the region of the ischial tuberosities.

The ischial tuberosity area for sitting on a foam cushion was, according to Aissaoui et al. (2001), a more or less circular area of about $71 \mathrm{~cm}^{2}$, or a diameter of $9.5 \mathrm{~cm}$. For sitting on a flat and hard surface this area is reduced. In this research we selected an area of $5 \times 5$ elements, which represents a square region of $6 \times 6 \mathrm{~cm}$.

As a matter of fact a detailed description of the pressure gradient as a function of $(x, y)$ in the region of interest gives the highest amount of information, but such is not practical. Therefore it was decided to apply data reduction so that the gradient distribution can be represented by one typical figure. This was elaborated by two methods. Around the ischial tuberosities the pressure gradient was computed according to the circular method and the transverse method. The circular method estimates an average of the magnitude of the gra- 


\section{Array for circular pressure gradient}

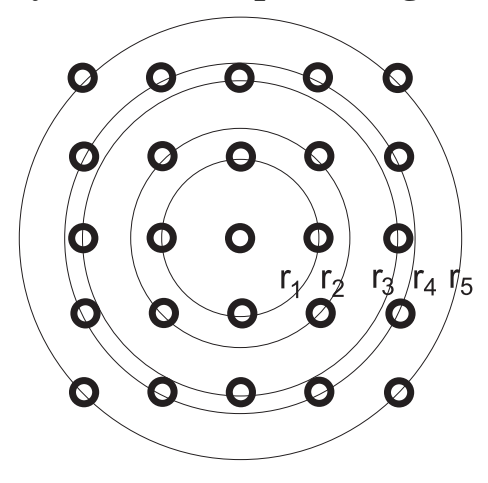

Figure 14: The array of $5 \times 5$ cells that was used to compute the circular pressure gradient.

dient over a square array pressure sensors of the pressure distribution measuring device. It assumes symmetry to the centre of the array. The transverse method estimates the gradient along a transversal line through the 'pressure mountains', and is obtained via measurements on the contourplots of the pressure distribution.

The circular gradient was computed for an area of $5 \times 5$ elements with the element of maximum pressure in the centre. First the gradient is computed for each of the contributing cells by fitting (least squares) a flat plane to an array of $3 \times 3$ elements with the current element in the center. Assuming the resulting normal vector $\vec{n}=\left(x_{n}, y_{n}, z_{n}\right)$, then the slope of this plane with a horizontal plane equals $\arctan \left(z_{n} / \sqrt{x_{n}^{2}+y_{n}^{2}}\right)$. This slope corresponds to the circular gradient of element $(i, j)$.

To compute the average gradient $G^{\prime}\left(r_{i}\right)$ along a circle with the main element in the centre, circles were drawn with the element of maximum pressure at the centre and the radius equal to the distance to the measuring cells, see figure 14. Thus five radii were obtained. The smallest radius equals the heart-to-heart distance of two neighbouring elements, $r_{1}=d$. In the pressure distribution measuring device $d=1.2 \mathrm{~cm}$. The other radii 
are $r_{2}=d \sqrt{2}, r_{3}=2 d, r_{4}=d \sqrt{5}$ and $r_{5}=2 d \sqrt{2}$. In this $5 \times 5$ array each circle has four elements, except radius 4 , which has eight elements. The result is five partial circular gradients:

$$
G^{C}\left(r_{1}\right)=\sum_{j=1, n_{1}} \frac{p_{m}-p_{r_{1, j}}}{r_{1}} \quad \ldots \quad G^{C}\left(r_{5}\right)=\sum_{j=1, n_{5}} \frac{p_{m}-p_{r_{5, j}}}{r_{5}}
$$

where $n_{j}$ is the number of elements of a specific circle. The final circular pressure gradient is defined as the average of these five circular gradients:

$$
G^{C}=\frac{1}{5} \Sigma G^{C}\left(r_{i}\right)
$$

The transverse gradient was obtained from the contourline plots of the pressure distribution at the lateral and the medial aspects of the maximum pressure areas, see figure 3. For each of the two pressure areas a line was drawn through the two maximum pressure points. Then the intersection points with the $10 \mathrm{kPa}$ contourlines were determined, lateral and medial to the maximum pressure points. Finally the distance between the medial and lateral intersection points, $\Delta s_{m, r i g h t}, \Delta s_{l, \text { right }}, \Delta s_{m, l e f t}$ and $\Delta s_{l, l e f t}$, was determined, see figure 3. The corresponding transverse pressure gradients, which were computed as $\Delta p / \Delta s$ are called $G_{l, \text { right }}^{T}, G_{m, \text { right }}^{T}, G_{m, l e f t}^{T}$ and $G_{l, l e f t}^{T}$. The average lateral and medial gradients and the total average transverse gradient are computed as:

$$
\begin{aligned}
G_{l}^{T} & =\frac{1}{2}\left(G_{T_{l, \text { right }}}+G_{T_{l, l e f t}}\right) \\
G_{m}^{T} & =\frac{1}{2}\left(G_{T_{m, \text { right }}}+G_{T_{m, l e f t}}\right) \\
G^{T} & =\frac{1}{2}\left(G_{l}^{T}+G_{m}^{T}\right)
\end{aligned}
$$




\section{References}

R Aissaoui, C Kauffmann, J Dansereau, and JA de Guise. Analysis of pressure distribution at the body-seat interface in able-bodies and paraplegic subjects using a deformable active contour algorithm. Medical Engineering and Physics, 23:369-367, 2001.

B Åkerblom. Standing and sitting posture. Nordiska Bokhandeln, Stockholm, 1948.

DL Bader and MB Hawken. Pressure Distribution under the Ischium of Normal Subjects. Journal of Biomedical Engineering, 8:353-357, 1986.

GW Batten and RF Walters. GPCP: A General Purpose Contouring Program. Calcomp, California Computer products, Inc, Anaheim, California, 1971.

DM Brienza and PE Karg. Seat Cushion Optimization: A Comparison of Interface Pressure and Tissue Stiffness Characteristics for Spinal Cord Injured and Elderly Patients. Archives of Physical Medical Rehabilitation, 79:388-394, april 1998.

DM Brienza, KC Chung, CE Brubaker, J Wang, TE Karg, and CT Lin. A System for the Analysis of Seat Support Surfaces Using Surface Shape Control and Simultaneous Measurement of Applied Pressures. IEEE Transactions on Rehabilitation Engineering, 4(2):103-113, june 1996.

JEL Carter and BH Heath. Somatotyping - development and applications. Cambridge University Press, 1990.

JD Chodera and M Lord. Paedobarographic Foot Pressure Measurements and their Applications. In PH Kenedi, editor, Proceedings of the Strathclyde bioengineering seminar 
on Disability, pages 173-181, August, 1978, University of Strathclyde, Glasgow, 1978. McMillan Press.

WW Chow and EI Odell. Deformations and Stresses in Soft Body Tissues of a Sitting Person. Journal of Biomedical Engineering, 100(may):79-87, 1978.

LB Dahlin, N Danielson, T Ehira, G Lundborg, and B Rydevik. Mechanical effects of compression of peripheral nerves. Journal of Biomechenical Engineering, 108:120-122, may 1986.

RK Daniel and B Faibisoff. Muscle Coverage of Pressure Points - The Role of Myocutaneous Flaps. Annals of Plastic Surgery, 8(6):446-452, june 1982.

N Diffrient, AR Tilley, and D Harman. Humanscale 7/8/9. MIT Pr, 1981. (No further bibl. data available).

JVGA Durnin and J Womersley. Body fat assessment from total body density and its estimation from skinfold thickness. British Journal of Nutrition, 21(21):681, 1974.

E Fox and CG Ulrich. SigmaScan \& SigmaScan Pro 2.0, Image. User's manual, Jandel Scientific Software Development, Jandel GmbH, Erkrath, Germany, 1995.

SL Garber and TA Krouskop. Body Build and Its Relationship to Pressure Distribution in the Seated Wheelchair Patient. Archives of Physical Medical Rehabilitation, 63:17-20, january 1982.

SL Garber, TA Krouskop, and RE Carter. A system for Clinically Evaluating Wheelchair Pressure-Relief Cushions. American Journal Occupational Therapy, 39(9):565-570, 1978. 
CM Gross, RS Goonetilleke, KK Menon, JCN Banaag, and CM Nair. The biomechanical assessment and prediction of seat comfort. In R Lueder and K Noro, editors, Hard facts about Machines: the Ergonomics of seating, chapter VII-18, pages 231-253. Taylor \& Francis, 1994.

K Helbig. Sitzdruckverteilung beim ungepolsterten Sitz (Sitting pressure distribution using uncushioned seat). Anthropologischer Anzieger, 36(3):194-202, march 1978.

JL Henderson, SH Price, ME Brandstater, and BR Mandac. Efficacy of Three Measures to Relieve Pressure in Seated Persons with Spinal Cord Injury. Archives of Physical Medical Rehabilitation, 75:535-539, 1994.

DA Hobson. Contributions of posture and deformity to the body-seat interface conditions of a person with spinal cord injuries. $\mathrm{PhD}$ thesis, University of Strathclyde, Glasgow, Scotland, 1988.

RR Houle. Evaluation of seat devices designed to prevent ischemic ulcers in paraplegic patients. Archives of Physical Medical Rehabilitation, 50:587-594, 1969.

TW Kernozek, A Amundson, J Hummer, and P Wilder. Effects of Body Mass Index on Seat Interface Pressures of Elderly that were Institutionalized. In Proceedings of the 24th Annual Meeting of the American Society of Biomechanics, University of Illinois at Chicago, july 19-22, 2000. Available on http://www.asb-biomech.org/onlineabs/2000.html.

A Kira. The bathroom. Viking Press, New York, 1976.

M Kosiak. Etiology of decubitus ulcers. Archives of Physical Medical Rehabilitation, 42: 19-29, 1961. 
TA Krouskop. A synthesis of the factors that contribute to pressure sore formation. Medical Hypotheses, 11:255-267, 1983.

SP Levine, RL Kett, and M Ferguson-Pell. Tissue Shape and Deformation Versus Pressure as a Characterization of the Seating Interface. Assistive Technology, 2(3):93-99, 1990.

G Lundborg, R Myers, and H Powell. Nerve compression injury and increased endoneurial fluid pressure: a "miniature compartment syndrome". Journal of Neurology, Neurosurgery, and Psychiatry, 46:1119-1124, 1983.

C Maltais, J Dansereau, R Aissaoui, and M Lacoste. Assessment of Geometric and Mechanical Parameters in Wheelchair Seating: A Variability Study. IEEE Transactions on Rehabilitation Research, 7(1):91-98, march 1999.

W Mayo-Smith and GVB Cochran. Wheelchair Cushion Modification: Device for Locating High Pressure Regions. Archives of Physical Medical Rehabilitation, 62:135-136, 1981.

GE Miller and JL Seale. The Mechanics of Terminal Lymph Flow. Journal of Biomedical Engineering, 107(november):376-380, 1985.

RT Minami, R Mills, and R Pardoe. Gluteus Maximus myocutaneous flaps for repair of pressure sores. Plastic and Reconstructive Surgery, 60(2):242-249, august 1977.

RJ Minns, RA Sutton, A Duffus, and R Mattinson. Underseat pressure distribution in the sitting spinal injury patient. Paraplegia, 22:297-304, 1984.

CCM Moes. Calibration of a Pressure Distribution Measuring Device. Technical report, Delft Univ. of Technol, Fac. of Ind. Design Engin, Delft, the Netherlands, 1999.

CCM Moes. Measuring the Tilt of the Pelvis. Ergonomics, 41(12):1821-1831, 1998. 
G Neff and K Fischer. Modular Seat-Shells and Standardized Manufacture of Individually Shaped Seats for the Severely Disabled - The Tubingen Experience. Clinical Prosthetics and Orthotics, 11(1):49-54, 1987.

CWJ Oomens, DH van Campen, and HJ Grootenboer. In Vitro Compression of a Soft Tissue Layer on a Rigid Foundation. J of Biomechanics, 20(10):923-935, 1987.

NR Reddy, GvB Cochran, and TA Krouskop. Interstitial Fluid Flow as a Factor in Decubitus Ulcer Formation. Journal of Biomechechanics, 14(12):879-881, 1981a.

NR Reddy, V Palmieri, and GvB Cochran. Subcutaneous interstitial fluid pressure during external loading. American Journal of Physiology, 240:R327-R329, 1981b.

S Riley and DL Bader. Biomechanical measurements of back shape and interface pressures in unsupported sitting. Clinical Biomechanics, 3(2):114-117, 1988.

EM Romanus. Microcirculatory reactions to controlled tissue ischaemia and temperature: a vital microscopic study on the hamsters' cheek pouch. In RM Kenedi and JM Cowden, editors, Bedsore Biomechanics, pages 79-82. University Park Press, London, 1976.

B Rydevik and C Nordborg. Changes in nerve function and nerve fibre structure induced by acute, graded compression. Journal of Neurology, Neurosurgery, and Psychiatry, 43: $1070-1082,1980$.

SG Souther, SD Carr, and LM Vistness. Wheelchair cushions to reduce pressure under bony promenences. Archives of Physical Medical Rehabilitation, 55:460-464, 1974.

HAM Staarink. Sitting Posture, Comfort and Pressure. PhD thesis, Delft University of Technology, Delft, the Netherlands, 1995. 
F Stumbaum. Experimentelle Untersuchung und mathematische Simulation der Sitzhaltung auf Arbeitsstühlen. $\mathrm{PhD}$ thesis, Lehrstuhl und Institut für Arbeitsphysiologie, Technische Universität München, 1983.

D Treaster. Measurement of seat pressure distributions. Human Factors, 29(5):563-575, 1987.

E van Engelen. Comfortonderzoek fietszadels. Master's thesis, Fac. of Industrial Design Engineering, Delft University of Technology, 1988. in dutch.

PL Williams, R Warwick, M Dyson, and LH Bannister, editors. Gray's Anatomy. Churchill Livingstone, Edinburgh, 37 edition, 1989.

D Zacharkow. Posture: Sitting, Standing, Chair Design and exercise. Charles C Thomas, Springfield, Ill, 1988. 